\title{
A lysine-cysteine redox switch with an NOS bridge regulates enzyme function
}

https://doi.org/10.1038/s41586-021-03513-3

Received: 16 September 2020

Accepted: 1 April 2021

Published online: 5 May 2021

Check for updates

\author{
Marie Wensien ${ }^{1,2,8}$, Fabian Rabe von Pappenheim ${ }^{1,2,8}$, Lisa-Marie Funk ${ }^{1,2}$, Patrick Kloskowski ${ }^{1,2}$, \\ Ute Curth ${ }^{3}$, Ulf Diederichsen ${ }^{4}$, Jon Uranga ${ }^{5}$, Jin $\mathrm{Ye}^{5}$, Pan Fang ${ }^{6}$, Kuan-Ting Pan ${ }^{6}$, \\ Henning Urlaub ${ }^{6,7}$, Ricardo A. Mata ${ }^{5}$, Viktor Sautner, ${ }^{1,2}$ \& Kai Tittmann ${ }^{1,2 \bowtie}$
}

Disulfide bonds between cysteine residues are important post-translational modifications in proteins that have critical roles for protein structure and stability, as redox-active catalytic groups in enzymes or allosteric redox switches that govern protein function ${ }^{1-4}$. In addition to forming disulfide bridges, cysteine residues are susceptible to oxidation by reactive oxygen species, and are thus central not only to the scavenging of these but also to cellular signalling and communication in biological as well as pathological contexts $s^{5,6}$. Oxidized cysteine species are highly reactive and may form covalent conjugates with, for example, tyrosines in the active sites of some redox enzymes ${ }^{7,8}$. However, to our knowledge, regulatory switches with covalent crosslinks other than disulfides have not previously been demonstrated. Here we report the discovery of a covalent crosslink between a cysteine and a lysine residue with a NOS bridge that serves as an allosteric redox switch in the transaldolase enzyme of Neisseria gonorrhoeae, the pathogen that causes gonorrhoea. X-ray structure analysis of the protein in the oxidized and reduced state reveals a loaded-spring mechanism that involves a structural relaxation upon redox activation, which is propagated from the allosteric redox switch at the protein surface to the active site in the protein interior. This relaxation leads to a reconfiguration of key catalytic residues and elicits an increase in enzymatic activity of several orders of magnitude. The redox switch is highly conserved in related transaldolases from other members of the Neisseriaceae; for example, it is present in the transaldolase of Neisseria meningitides (a pathogen that is the primary cause of meningitis and septicaemia in children). We surveyed the Protein Data Bank and found that the NOS bridge exists in diverse protein families across all domains of life (including Homo sapiens) and that it is often located at catalytic or regulatory hotspots. Our findings will inform strategies for the design of proteins and peptides, as well as the development of new classes of drugs and antibodies that target the lysine-cysteine redox switch ${ }^{9,10}$.
The three-dimensional structures of proteins are formed and maintained by a delicate balance of stabilizing and destabilizing forces that are mostly of a noncovalent nature, such as hydrogen bonds, van der Waals forces and charge-charge interactions. In addition to peptide bonds, only a few additional covalent bonds between amino acids contribute to the structure, stability and function of proteins. Major classes of these bonds include disulfide bonds between two cysteines (which are the most prevalent post-translational modification in proteins) and isopeptide bonds between lysine and asparagine or glutamine residues ${ }^{2-5,11}$. Although both of these types of bond affect the fold and stability of proteins, disulfide bonds are unique in their ability to undergo reversible bond cleavage by redox chemistry to form the corresponding dithiol, either as part of the enzymatic reaction mechanism or as an allosteric switch that changes the structure (and with that the function) of a given protein ${ }^{4,12}$ (Extended Data Fig. 1a, b). In addition to disulfides, a variety of other oxidized cysteine species in different oxidation states serve as molecular sensors, triggers and scavengers in numerous cellular processes including signalling, communication and stress response $\mathrm{e}^{5,6}$.

\section{Allosteric redox switch in a transaldolase}

Because of our interest in the structure and mechanism of transaldolase $(\mathrm{TAL})^{13,14}$, which is a key enzyme in the pentose phosphate pathway in all

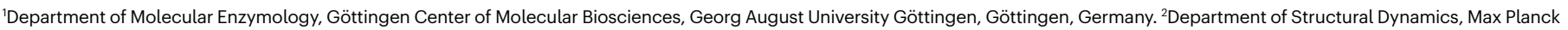

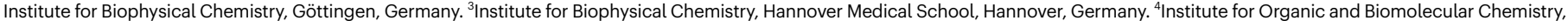

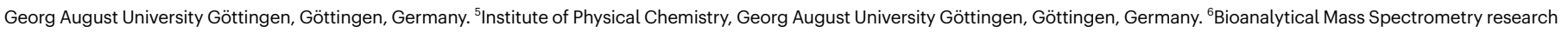
group, Max Planck Institute for Biophysical Chemistry, Göttingen, Germany. ${ }^{7}$ University Medical Center Göttingen, Bioanalytics, Institute for Clinical Chemistry, Göttingen, Germany. ${ }^{8}$ These authors contributed equally: Marie Wensien, Fabian Rabe von Pappenheim. ${ }^{\circledR}$ e-mail: ktittma@gwdg.de 
domains of life, we initiated studies of the orthologue from N. gonorrhoeae (NgTAL). We aimed to devise mechanism-inspired inhibitors as drugs for treating gonorrhoea, a sexually transmitted disease of which there are approximately 100 million cases worldwide. Gonorrhoea is typically treated with antibiotics, but increasing rates of antibiotic resistance pose a serious threat ${ }^{15}$. When produced recombinantly, NgTAL showed almost no enzymatic activity. We hypothesized that the enzyme could be subject to activation by a redox event that involves one or more of its three cysteine residues: Cys38, Cys87 and Cys90. We thus tested whether the activity of $\mathrm{NgTAL}$ is redox-regulated by a disulfide-dithiol switch or by oxidation of one of these cysteines. Cys38, Cys87 and Cys90 are located in close proximity to one another but are not engaged in disulfide linkage(s) in a previous X-ray structure deposited in the Protein Data Bank (PDB) (code 3CLM; www.rcsb. org) (Fig. 1a). When we added reductants such as dithiothreitol or tris(2-carboxyethyl)phosphine to the protein, enzymatic activity was increased by several orders of magnitude, which affected both substrate binding (decreased Michaelis constant $\left(K_{\mathrm{M}}\right)$ ) and catalysis (increased catalytic constant $\left(k_{\text {cat }}\right)$ ) (Fig. $1 b$, Extended Data Table 1$)$. This redox activation is essentially reversible: the reduced enzyme converts into the inactive, oxidized form over the course of several days at $6^{\circ} \mathrm{C}$, and can be re-activated by re-reduction (Supplementary Fig. 1). The overall structure of the enzyme, as judged by far-ultraviolet circular dichroism (far-UV CD) spectroscopy, is not altered upon reduction (Fig. 1c). However, our thermal unfolding analyses using far-UV CD spectroscopy revealed differences for the oxidized and reduced protein. The reduced protein exhibited a highly cooperative unfolding, whereas the oxidized protein displayed a less pronounced cooperativity of unfolding and an increased melting temperature (Fig. 1d). To determine which of the three cysteines in $\mathrm{NgTAL}$ is involved in redox regulation, we constructed single-site variants that lacked each of them. We predicted that the substitution of a cysteine that belongs to the redox switch would result in a regulation 'knockout' and nullify redox activation. Of the three cysteine variants we tested, only a variant with a substitution of Cys38 (Cys38Ser) exhibited a loss of redox activation. This variant was constitutively active in both redox states, whereas variants with substitutions of the two other cysteines (Cys87 and Cys90) retained the ability to become activated by reduction (Extended Data Table 1). This excludes the presence of an intramolecular disulfide; we therefore hypothesized that Cys38 might form an intermolecular disulfide bridge with its counterpart from another protein chain in a multimeric assembly, and that redox activation is coupled to disassembly of the less active oligomer (which represents a mechanism that has previously been reported for other proteins that bear allosteric disulfide redox switches ${ }^{16}$ ). We then used analytical ultracentrifugation to assess the oligomerization state of $\mathrm{NgTAL}$ under oxidizing and reducing conditions. The enzyme was predominantly a monomer under all of the conditions we tested, which falsified our hypothesis that it contained a redox switch that involved a change in quaternary structure (Fig. 1e). This finding suggested that there is an intramolecular linkage in the oxidized protein. As only one of the three cysteine residues in $\mathrm{NgTAL}$ is functionally important for redox regulation, this raises questions about the chemical nature of the redox switch.

\section{Discovery of a lysine-cysteine redox switch}

To further investigate the basis for redox activation, we crystallized $N g$ TAL under oxidizing and reducing conditions. We determined structures of both states at a true atomic resolution of $0.96 \AA$ for the oxidized enzyme, and $1.05 \AA$ for the reduced enzyme (crystallographic statistics are provided in Extended Data Table 2). In the oxidized state, we observed a crosslink between Cys38 and Lys8 that contained an additional, unidentified atom $(X)$ that covalently bridged the sulfur atom of cysteine and the nitrogen atom of lysine (Fig. 2a). We reproducibly observed this crosslink in numerous protein crystals that were
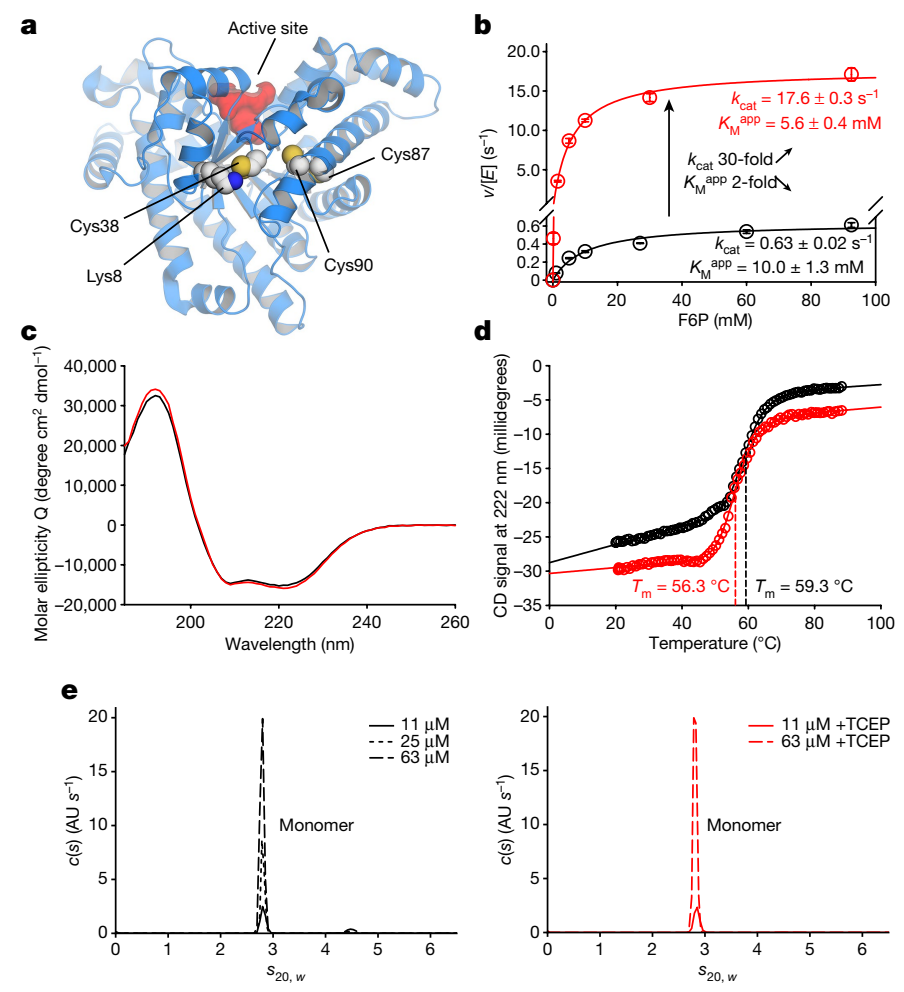

Fig. 1 | Functional and structural analysis of $\mathrm{NgTAL}$ in the oxidized and reduced state. a, Crystallographic structure of $\mathrm{NgTAL}$ (PDB code 3CLM), showing the positions of residues Lys 8, Cys38, Cys 87 and Cys90, as well as the active site (highlighted in red).b, Steady-state kinetic analysis of enzymatic activity of $\mathrm{NgTAL}$ in the oxidized (black) and reduced (red) state. There is a 30 -fold increase of $k_{\text {cat }}$ and a 2 -fold decrease of substrate $K_{\mathrm{M}}$ upon reduction. All measurements were carried out in triplicate and are shown as mean \pm s.d. Kinetic and thermodynamic constants are provided in Extended Data Table1. Experiments were repeated three times with identical results. F6P, fructose 6-phosphate. c, Far-UV CD spectra of NgTAL in the oxidized (black) and reduced (red) state.d, Thermal unfolding of NgTAL in the oxidized (black) and reduced (red) state, monitored by near-UV CD spectroscopy at $222 \mathrm{~nm}$. The less cooperative unfolding and increased melting temperature $\left(T_{\mathrm{m}}\right)$ of the oxidized protein suggest the presence of a covalent crosslink. e, Analytical ultracentrifugation of $\mathrm{NgTAL}$ in the oxidized (left) and reduced (right) state at different concentrations of protein. For reduction, $1 \mathrm{mM}$ tris(2-carboxyethyl) phosphine (TCEP) was used. Independent of protein concentration and presence of TCEP, an $s$ value of 2.8 and a molar mass of $37.1 \mathrm{~kg} \mathrm{~mol}^{-1}$ were obtained from $c(s)$ analysis. Because the molar mass of $\mathrm{NgTAL}$ calculated from the amino acid sequence is $37.6 \mathrm{~kg} \mathrm{~mol}^{-1}$, the protein is monomeric in solution. The very small percentage of material that sediments at an $s$ value of about 4.5 disappears in the presence of reducing agent, which indicates the presence of some incorrectly linked dimer. AU, arbitrary unit.

obtained under different, nonreducing crystallization conditions, as well as in a low-dose dataset that was collected from an in-house rotating anode (which excluded the possibility that formation of the crosslink resulted from 'radiation damage' during data collection at the high-energy synchrotron beamline) (Extended Data Fig. 1c-f). To reveal the identity of atom $\mathrm{X}$, we performed competitive refinements, which-at sub-Ångstrøm resolution-enable different types of atom to be reliably discerned ${ }^{17}$. We considered two possibilities: (1) a bridging oxygen atom that originates from cysteine and/or lysine oxidation or (2) a bridging carbon atom, because methylene bridges between lysines and cysteines have previously been reported to form in consequence of the exposure of protein crystals to formaldehyde and/or carbon dioxide (although this atom assignment has been questioned $)^{18,19}$. In our case, the bridging atom $X$ was clearly identified as an oxygen atom: refinements using carbon at this bridging position 


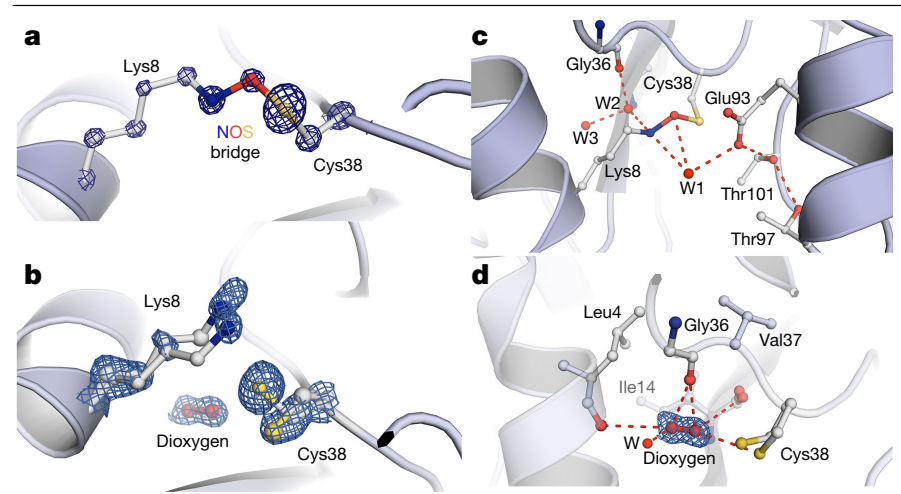

Fig. 2 | Structure of the allosteric redox switch in $\mathbf{N g T A L}$ in the oxidized and reduced state. a, Structure of the Lys $8-C y s 38$ crosslink in the oxidized state, containing a covalent NOS bridge with an oxygen atom that links the lysine and cysteine side chains. The refined structural model is superposed with the $2 m F_{0}-D F_{c}$ electron density map (shown in blue) contoured at $3 \sigma$. b. Structure of the redox switch in the reduced state. The covalent NOS bridge between Lys 8 and Cys 38 is disengaged. Both residues are structurally disordered and exhibit several conformations. We observed electron density that is compatible with a physically dissolved dioxygen molecule in close vicinity to the redox switch. This feature was not present in the oxidized state. The structural model is superposed with the $2 m F_{\mathrm{o}}-D F_{\mathrm{c}}$ electron density map (shown in blue) contoured at $1.5 \sigma$.c Local environment of the Lys8-Cys38 bridge in oxidized $\mathrm{NgTAL}$, showing hydrogen-bonding interactions with water molecules $(\mathrm{W})$ and protein groups.d, Binding locale of the presumed dioxygen molecule in reduced $N g \mathrm{TAL}$, showing hydrogen-bonding interactions with water molecules and protein groups. The dioxygen molecule is close to Cys 38 , which suggests that formation of the Lys8-Cys38 bridge probably results from initial cysteine oxidation.

resulted in unexplained positive difference peaks in the electron density maps (Extended Data Fig. 2c). The two remaining cysteines (Cys87 and Cys90) are present in the thiol form. These results demonstrate that in the oxidized state the inactive $\mathrm{NgTAL}$ enzyme contains a covalent lysine-cysteine crosslink with an NOS bridge. The NOS arrangement resembles that of hydroxylamine- $O$-sulfonic $\operatorname{acid}^{20}$ (an organic reagent used for amination), although in this acid the sulfur atom is in a higher oxidation state than it is in the enzyme.

In the structure of $\mathrm{NgTAL}$ in the reduced state (cocrystallized in presence of the reductant $\beta$-mercaptoethanol), the covalent lysine-cysteine NOS bridge is absent. Both Lys 8 and Cys 38 are chemically unmodified and show several alternative conformations (Fig. 2b). We observed electron density in close proximity to the sulfur atom of Cys38 that is compatible with a physically dissolved dioxygen molecule, which suggests that oxidation of this cysteine by molecular oxygen precedes the formation of the covalent crosslink. The NOS bridge is located at the protein surface and is accessible to solvent. It is engaged in hydrogen-bond interactions with several water molecules as part of an extended hydrogen-bonding network that further includes the residues Glu93, Thr97 and Thr101, which suggests a role for these residues in the formation of the NOS linkage (Fig. 2c). The presumed dioxygen is bound in a small hydrophobic patch that is close to Ile14 and within hydrogen-bonding distance of Cys38, and is coordinated by several interactions with the main chain of the protein (Fig. 2d). This dioxygen is not observed in oxidized NgTAL with the NOS bridge (Extended Data Fig. 1g).

To define the chemical basis of the formation of the NOS bridge, we studied the individual reactivities of Cys 38 and Lys 8 in the absence of the corresponding NOS bridge partner. We analysed the structure and function of the variants Lys8Ala (in which Cys 38 of the NOS bridge is present) and Cys38Ser (in which Lys 8 of the NOS bridge is present) (Extended Data Fig. 2, Extended Data Table 1). The variant Cys38Ser is not activated by reduction and shows identical melting temperatures for unfolding under oxidizing and reducing conditions, whereas the variant Lys8Ala is subject to redox activation and exhibits altered melting temperatures upon redox activation. This suggests that the Cys38 in the variant Lys8Ala is subject to a chemical modification (most probably an oxidation), whereas Lys8 in variant Cys38Ser is probably unmodified. To test this hypothesis, we determined the X-ray crystal structures of the variants at a resolution of $0.85 \AA$ (for the Lys 8 Ala variant) and $1.25 \AA$ (for the Cys38Ser variant) (Extended Data Fig. 2c, d, Extended Data Table 2). These structural data support our notion that the Cys 38 in the Lys8Ala variant is oxidized, whereas the Lys8 in the Cys38Ser variant is chemically unmodified. These observations bolster our claim that the Cys38-Lys 8 crosslink results from an oxidation reaction, which is further supported by mass spectrometry analysis ('Results' in Supplementary Information, Supplementary Fig. 2).

\section{Mechanism of NOS bridge formation}

Despite the unambiguous structural information, it is difficult at this point to determine the chemical mechanism of the formation of the NOS linkage-especially as, to our knowledge, there is no precedent for this reaction in organic or protein chemistry. In principle, oxidized cysteines could react with amines; however, the nucleophilic nitrogen would preferentially attack the sulfur atom instead of a sulfur-bound oxygen, to produce sulfonamides or sulfinamides ${ }^{21}$. We envisage several different mechanisms for the formation of the NOS bridge. First, the cysteine thiolate could react with dioxygen (or another reactive oxygen species) to yield a thio-(hydro)peroxy species that in turn reacts with the $\varepsilon$-amino group of the neighbouring Lys8 (Extended Data Fig. 3a). This reaction would directly afford the covalent NOS bridge, with concomitant water release facilitated by synchronized proton transfer from the attacking amine to the water-leaving group. In an alternative scenario (Extended Data Fig. 3b), oxygen or reactive oxygen species might oxidize both the cysteine thiol and the lysine amine to form sulfenic acid and hydroxylamine or amine oxide (either in a concerted reaction with a transient thio-(hydro)peroxy species or in independent reactions). Both hydroxylamine and amine oxide are potent $\mathrm{O}$-nucleophiles ${ }^{22}$ that would have sufficient reactivity to covalently react with an oxidized cysteine. Formation of the NOS bridge would then occur simultaneously with water elimination. In a third mechanism (Extended Data Fig. 3c), the lysine amine would first attack the sulfur atom of the previously oxidized cysteine (either sulfenic or sulfinic acid), followed by sigmatropic [1,2] rearrangement driven by orbital steering and stereoelectronic control. For this to happen, the enzyme would need to destabilize the stable $\mathrm{N}-\mathrm{S}$ bond of the presumed sulfinamide, and steer the orbitals to promote a [1,2] rearrangement (for example, by exerting strain) ${ }^{23,24}$.

We carried out electronic structure calculations to compare the different mechanistic hypotheses. We performed these calculations on the basis of a cluster model that included Lys8, Cys38 and Glu93, as well as four explicit water molecules to retain a hydrogen-bond network close to the reaction site. A schematic of the computed mechanisms and free energies is shown in Extended Data Fig. 3d. We started our investigations with the thio-(hydro)peroxy species. We identified a transition state for $\mathrm{O}-\mathrm{O}$ bond breakage and the concerted oxidation of Lys8. This step has an activation barrier of $12.2 \mathrm{kcal} \mathrm{mol}^{-1}$, and leads to the formation of sulfenic acid and the oxidized lysine. We observe that several different protonation states are accessible at this point, with the neighbouring water mediating proton transfer along the residues. We argue that further oxidation of the Cys 38 to sulfinic acid is not viable in the formation of the NOS bridge, because sulfinic acid is far too stable ( $41.4 \mathrm{kcal} \mathrm{mol}^{-1}$ lower in energy relative to the NOS linkage) and would effectively trap the reaction. A potential sigmatropic [1,2] rearrangement should also be excluded, as any pathway that involves a sulfinamide intermediate would result in barriers in excess of $35 \mathrm{kcal}$ $\mathrm{mol}^{-1}$. Considering the stability and the cysteine oxidation state in 


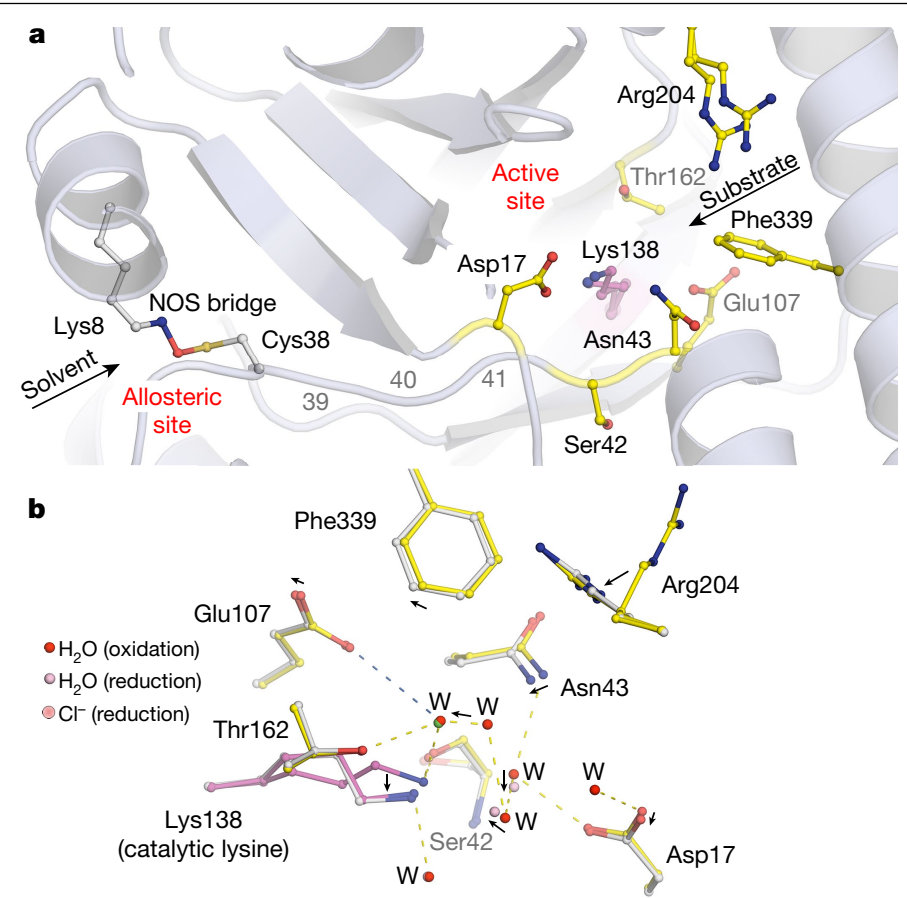

Fig. 3 | Structural basis of redox activation of $\mathbf{N g T A L}$. a, Structure of $\mathrm{NgTAL}$ in the oxidized state, showing the allosteric Lys8-Cys38 redox switch (in grey) at the protein surface and the active site (in yellow), including the cataly tic Lys138 that forms a Schiff base (highlighted in magenta). Cys38 is located on a strand-like structure that extends from the allosteric site to the active site, and which bears residues Ser42 and Asn43. The entrance to the active site and allosteric redox site are highlighted. b, Superposition of $\mathrm{NgTAL}$ in the oxidized (this Article (yellow)) and reduced (PDB code 3CLM (grey)) state, showing the subtle repositioning of active-site residues and water molecules upon redox activation. A superposition of the oxidized state with our structure of the reduced state that contains a covalent Schiff-base intermediate analogue is shown in Extended Data Fig. 8.

sulfinamide and in sulfinic acid, the mechanism for the formation of the NOS bridge must necessarily involve a lower oxidation state (for example, sulfenic acid). At this point, our quantum chemical calculations favour the second scenario outlined above.

\section{Mechanism of allosteric activation}

To understand the structural basis for the decrease in NgTAL activity upon formation of the NOS linkage, we superposed the oxidized and reduced structures (Fig. 3). Notably, Cys38 of the redox switch is located on an extended strand-like structure that reaches from the protein surface into the active site and bears the active-site residues Ser42 and Asn43 (Fig. 3a). The backbone oxygen of Ser42 forms a hydrogen bond with the $\varepsilon$-amino group of the catalytic Schiff-base-forming Lys138 and Asn43 is a critical active-site residue that has roles in substrate binding and catalysis ${ }^{13,25}$. Reduction of the cysteine-lysine NOS redox switch entails a slight structural repositioning of the Cys38-bearing strand (which includes interacting strands and helices) that leads to a slightly reconfigured active site, in which the key catalytic residues Asn 43 and Asp17, as well as catalytic water molecules, are repositioned (Fig. $3 b$ ). Although we found that the catalytic Lys138 is disordered in the oxidized, inactive state, it becomes ordered upon reduction and activation. It is known for this enzyme, and for many other classes of enzyme, that orbital steering is critical for catalysis, and that small structural changes can lead to large changes in catalytic competence $\mathrm{c}^{24,26,27}$.

\section{Conservation of NOS bridges in TALs}

We next asked whether the observed NOS bridge is unique to $\mathrm{NgTAL}$ or whether it is potentially conserved in related orthologues. We hypothesized that a potential redox regulation mechanism with in vivo relevance is very likely to be conserved, and not to be a unique feature of a single protein. Our genetic analyses revealed the residues Lys 8 and Cys 38 (which form the NOS bridge) are highly conserved in the TALs of members of the Neisseriaceae, and also in those of other clades (for example, Cyanophyceae) (Extended Data Fig. 4). As a proof of concept, we studied the TAL from N. meningitidis (NmTAL), which is the main cause of bacterial meningitis and septicaemia in children and young adults. This enzyme is highly similar to $\mathrm{NgTAL}$, and contains a lysine and cysteine at equivalent positions to those that form the NOS bridge in NgTAL. Our functional and structural analyses of $N m$ TAL revealed that this enzyme is subject to redox activation (Extended Data Fig. 5, Extended Data Table 1). Besides the effect on enzymatic activity, we observed that the oxidized enzyme in which the NOS bridge is likely to be present exhibits notable thermal stability and is not completely unfolded. Despite the high degree of similarity of $N m$ TAL and $N g$ TAL at a sequence level, the enzymatic activity of the two enzymes in the oxidized state is different, which suggests subtle crosstalk between the allosteric and active site. The high degree of conservation of NOS-bridge redox switches in NgTAL and $N m$ TAL suggests that the formation of this bridge is related to the stress response to reactive oxygen species, as these two organisms are human pathogens and encounter oxidative stress as a host defence mechanism ${ }^{28,29}$. The formation of the NOS bridge in the TALs would change the metabolic flux through the pentose phosphate pathway, and thus affect ribose production for the biosynthesis of nucleic acids.

\section{Analysis of structures from the PDB}

We next asked whether the cysteine-lysine NOS bridge exists in other proteins. We searched the PDB for structures that contained juxtaposed lysine and cysteine side chains with unexplained electron density between the sulfur atom of the cysteine and the nitrogen atom of the lysine. Unambiguous identification of the NOS bridge requires structures at true atomic resolution, as well as sufficient occupancy. As this chemical entity was previously unknown, it could have easily been disregarded in previous interpretations of electron density maps. When searching the PDB, we identified a number of proteins from all domains of life that are highly likely to possess one or more NOS bridges. We identified several chemical and structural motifs, which will be discussed in a separate publication (F.R.v.P.et al., manuscript in preparation). Here we discuss in brief three representative examples in which the NOS bridges are likely to have an effect upon the biological function of the protein. In the human protein 8-oxoguanine glycosylase OGG1 (which is involved in DNA base excision repair ${ }^{30}$ under oxidative stress conditions, and is implicated in several types of cancer $^{31}$ ), an NOS bridge is observed between residues Cys253 and Lys249 (Extended Data Fig. 6). As residue Lys 249 is the key catalytic residue that forms a Schiff-base linkage with the DNA, formation of the NOS bridge-if relevant in vivo-could potentially regulate the enzymatic activity (for example, through inactivation of the essential lysine). NOS bridges are also found at the substrate-binding locale of the active site of enzymes, or at a critical position at the binding interface of proteins that form the nuclear egress complex of herpesviruses and thus potentially regulate substrate or protein binding (Extended Data Fig. 7).

\section{Conclusions}

Here we report on a redox switch in proteins that contains a lysinecysteine crosslink with a covalent NOS bridge. Our analysis shows 


\section{Article}

that the NOS bridge is not unique to $N g T A L$, but that it may instead contribute to the function, structure and stability of a diverse set of proteins. The spatial constraints for forming an NOS bridge are not as tight as those for a disulfide bond, as the lysine side chain is larger than that of a cysteine and thus allows for more flexibility in tethering protein chains intra- and intermolecularly. The involvement of lysine as part of the redox switch expands the regulatory tool set from that of disulfide-dithiol switches. Our quantum chemical calculations indicate that the NOS bridge is of high energy, which ensures reversibility. This finding may serve as a springboard for peptide and protein engineering by implementing the lysine-cysteine motif into natural or designer proteins ${ }^{32}$, as well as for drug design by developing small-molecule inhibitors $^{10,33}$, antibodies and nanobodies (or conjugates of these) that target the switch, given that the detected proteins are in part from pathogenic bacteria or viruses ${ }^{34,35}$. The possibility that neighbouring lysine and cysteine residues may form NOS bridges should also be implemented in tools for structure prediction ${ }^{36,37}$.

\section{Online content}

Any methods, additional references, Nature Research reporting summaries, source data, extended data, supplementary information, acknowledgements, peer review information; details of author contributions and competing interests; and statements of data and code availability are available at https://doi.org/10.1038/s41586-021-03513-3.

1. Bardwell, J. C., McGovern, K. \& Beckwith, J. Identification of a protein required for disulfide bond formation in vivo. Cell 67, 581-589 (1991)

2. Wedemeyer, W. J., Welker, E., Narayan, M. \& Scheraga, H. A. Disulfide bonds and protein folding. Biochemistry 39, 4207-4216 (2000).

3. Matsumura, M. \& Matthews, B. W. Control of enzyme activity by an engineered disulfide bond. Science 243, 792-794 (1989).

4. Hogg, P. J. Disulfide bonds as switches for protein function. Trends Biochem. Sci. 28 210-214 (2003).

5. Paulsen, C. E. \& Carroll, K. S. Cysteine-mediated redox signaling: chemistry, biology, and tools for discovery. Chem. Rev. 113, 4633-4679 (2013).

6. Depuydt, M. et al. A periplasmic reducing system protects single cysteine residues from oxidation. Science 326, 1109-1111 (2009).

7. Ito, N. et al. Novel thioether bond revealed by a $1.7 \AA$ A crystal structure of galactose oxidase. Nature 350, 87-90 (1991).

8. Firbank, S. J. et al. Crystal structure of the precursor of galactose oxidase: an unusual self-processing enzyme. Proc. Natl Acad. Sci. USA 98, 12932-12937 (2001).

9. Dror, R. O. et al. Structural basis for modulation of a G-protein-coupled receptor by allosteric drugs. Nature 503, 295-299 (2013).

10. Nussinov, R. \& Tsai, C. J. The design of covalent allosteric drugs. Annu. Rev. Pharmacol. Toxicol. 55, 249-267 (2015).

11. Kang, H. J., Coulibaly, F., Clow, F., Proft, T. \& Baker, E. N. Stabilizing isopeptide bonds revealed in Gram-positive bacterial pilus structure. Science 318, 1625-1628 (2007).

12. Uhlin, U. \& Eklund, H. Structure of ribonucleotide reductase protein R1. Nature 370, 533-539 (1994).
13. Lehwess-Litzmann, A. et al. Twisted Schiff base intermediates and substrate locale revise transaldolase mechanism. Nat. Chem. Biol. 7, 678-684 (2011).

14. Tittmann, K. Sweet siblings with different faces: the mechanisms of FBP and F6P aldolase, transaldolase, transketolase and phosphoketolase revisited in light of recent structural data. Bioorg. Chem. 57, 263-280 (2014).

15. Wi, T. et al. Antimicrobial resistance in Neisseria gonorrhoeae: global surveillance and a call for international collaborative action. PLoS Med. 14, e1002344 (2017).

16. Chiu, J. \& Hogg, P. J. Allosteric disulfides: sophisticated molecular structures enabling flexible protein regulation. J. Biol. Chem. 294, 2949-2960 (2019).

17. Neumann, P. \& Tittmann, K. Marvels of enzyme catalysis at true atomic resolution: distortions, bond elongations, hidden flips, protonation states and atom identities. Curr. Opin. Struct. Biol. 29, 122-133 (2014).

18. Ruszkowski, M. \& Dauter, Z. On methylene-bridged cysteine and lysine residues in proteins. Protein Sci. 25, 1734-1736 (2016).

19. Wang, J. Crystallographic identification of spontaneous oxidation intermediates and products of protein sulfhydryl groups. Protein Sci. 28, 472-477 (2019).

20. Kovacic, P. \& Bennett, R. P. Aromatic amination with hydroxylamine-O-sulfonic acid. J. Am. Chem. Soc. 83, 221-224 (1961).

21. Akter, S. et al. Chemical proteomics reveals new targets of cysteine sulfinic acid reductase. Nat. Chem. Biol. 14, 995-1004 (2018).

22. Kirby, A. J. et al. Hydroxylamine as an oxygen nucleophile. Structure and reactivity of ammonia oxide. J. Am. Chem. Soc. 128, 12374-12375 (2006).

23. Lüdtke, S. et al. Sub-Ångström-resolution crystallography reveals physical distortions that enhance reactivity of a covalent enzymatic intermediate. Nat. Chem. 5, 762-767 (2013).

24. Mesecar, A. D., Stoddard, B. L. \& Koshland, D. E. Jr. Orbital steering in the catalytic power of enzymes: small structural changes with large catalytic consequences. Science $\mathbf{2 7 7}$ 202-206 (1997).

25. Schörken, U. et al. Identification of catalytically important residues in the active site of Escherichia coli transaldolase. Eur. J. Biochem. 268, 2408-2415 (2001).

26. Dai, S. et al. Low-barrier hydrogen bonds in enzyme cooperativity. Nature $\mathbf{5 7 3}, 609-613$ (2019).

27. Rabe von Pappenheim, F. et al. Structural basis for antibiotic action of the $B_{1}$ antivitamin 2'-methoxy-thiamine. Nat. Chem. Biol. 16, 1237-1245 (2020).

28. Seib, K. L. et al. Defenses against oxidative stress in Neisseria gonorrhoeae: a system tailored for a challenging environment. Microbiol. Mol. Biol. Rev. 70, 344-361 (2006).

29. Seib, K. L., Tseng, H. J., McEwan, A. G., Apicella, M. A. \& Jennings, M. P. Defenses against oxidative stress in Neisseria gonorrhoeae and Neisseria meningitidis: distinctive systems for different lifestyles. J. Infect. Dis. 190, 136-147 (2004).

30. Bruner, S. D., Norman, D. P. \& Verdine, G. L. Structural basis for recognition and repair of the endogenous mutagen 8-oxoguanine in DNA. Nature 403, 859-866 (2000).

31. Chevillard, S. et al. Mutations in OGG1, a gene involved in the repair of oxidative DNA damage, are found in human lung and kidney tumours. Oncogene 16, 3083-3086 (1998).

32. Huang, P. S., Boyken, S. E. \& Baker, D. The coming of age of de novo protein design. Nature 537, 320-327 (2016).

33. Singh, J., Petter, R. C., Baillie, T. A. \& Whitty, A. The resurgence of covalent drugs. Nat. Rev. Drug Discov. 10, 307-317 (2011).

34. Scott, A. M., Wolchok, J. D. \& Old, L. J. Antibody therapy of cancer. Nat. Rev. Cancer 12 278-287 (2012).

35. Muyldermans, S. Nanobodies: natural single-domain antibodies. Annu. Rev. Biochem. 82, 775-797 (2013).

36. Senior, A. W. et al. Improved protein structure prediction using potentials from deep learning. Nature 577, 706-710 (2020).

37. Callaway, E. 'It will change everything': DeepMind's Al makes gigantic leap in solving protein structures. Nature 588, 203-204 (2020).

Publisher's note Springer Nature remains neutral with regard to jurisdictional claims in published maps and institutional affiliations.

(c) The Author(s), under exclusive licence to Springer Nature Limited 2021 


\section{Methods}

No statistical methods were used to predetermine sample size. The experiments were not randomized, and investigators were not blinded to allocation during experiments and outcome assessment.

\section{Experimental procedures}

General information. The protein concentration of $\mathrm{NgTAL}$ was determined via UV and visible light spectroscopy by measuring absorption at $280 \mathrm{~nm}$, using the molar extinction coefficient $\left(\varepsilon_{N g \mathrm{TAL}}=28,420 \mathrm{M}^{-1} \mathrm{~cm}^{-1}\right)$, which was determined according a previous publication ${ }^{38}$.

Cloning and expression. The gene that encodes NgTAL (UniProt identifier Q5F6E9) was chemically synthesized by GeneArt, Invitrogen (Thermo Fisher Scientific) with an additional $\mathrm{N}$-terminal glycine according to a previously deposited structure (PDB code 3CLM), and recloned into the $\mathrm{pET}$ SUMO vector via Champion $\mathrm{PET}$ SUMO TA Cloning Kit (Thermo Fisher Scientific) using the subcloning primers: forward 5'-GGTATGACCATTCTGAGTGATG-3'; and reverse 5'-TTAAACCAGCGGTGCCAGC-3'.

Subsequently, mutant strains were generated by site-directed mutagenesis PCR using the QuikChange site-directed mutagenesis protocol (Stratagene). The following primer pairs were used: for the Lys8Ala variant, forward 5'-GAGTGATGTTGCAGCACTGGGTCAGC-3' and reverse 5'-GCTGACCCAGTGCTGCAACATCACTC-3'; for the Cys38Ser variant, forward $5^{\prime}$-GCTGAAACAGGGTGTTTCTGGTGTTACCAGC-3' and reverse 5'-GCTGGTAACACCAGAAACACCCTGTTTCAGC-3'; for the Cys87Ala variant, forward 5'-CGTGCAGCAGCTGATGTTTGTCTGG-3' and reverse 5'-CCAGACAAACATCAGCTGCTGCACG-3'; and for the Cys90Ala variant, forward $5^{\prime}$-GCATGTGATGTTGCTCTGGCAGAACATGAAAGC-3' and reverse 5'-GCTTTCATGTTCTGCCAGAGCAACATCACATGC-3'.

For recombinant expression, the vector pET SUMO containing the gene encoding $N g$ TAL was transformed into BL21 Star (DE3) One Shot chemically competent Escherichia coli cells (Thermo Fisher Scientific) according to a previous publication ${ }^{39}$. The bacteria were grown in LB medium ${ }^{40}$ containing $50 \mu \mathrm{g} \mathrm{ml}^{-1}$ kanamycin sulfate at $37^{\circ} \mathrm{C}$ until an optical density at $600 \mathrm{~nm}$ of $0.5-0.8$ was reached. Subsequently, gene expression was induced by addition of $200 \mu \mathrm{M}$ isopropyl- $\beta$-D-thio-galactopyranoside for about $20 \mathrm{~h}$ at $37^{\circ} \mathrm{C}$. The cells were collected by centrifugation at $5,750 \mathrm{~g}$, and either directly used or flash-frozen in liquid nitrogen and stored until use at $-80^{\circ} \mathrm{C}$.

The gene encoding NmTAL (UniProtID: X5EUF7) was chemically synthesized by GeneArt, Invitrogen (Thermo Fisher Scientific) as a pET-SUMO vector (pET28a-His6-SUMO-NmTAL).

\section{Protein purification}

All purification steps were performed at $4{ }^{\circ} \mathrm{C}$ or on ice. Cells were resuspended in buffer $\mathrm{A}(100 \mathrm{mM}$ Tris/ $\mathrm{HCl} \mathrm{pH} \mathrm{7.5,40} \mathrm{mM} \mathrm{imidazole}$ and $300 \mathrm{mM} \mathrm{NaCl}$ ), supplemented with $510 \mu \mathrm{M}$ phenylmethanesulfonyl fluoride, $0.5 \mathrm{mg} \mathrm{m}^{-1}$ lysozyme (AppliChem), $5 \mathrm{mM} \mathrm{MgCl}_{2}$ and $5 \mu \mathrm{g} \mathrm{ml}^{-1}$ DNasel (Thermo Fisher Scientific), and subsequently lysed by five passages through a LM10 Microfluidizer High Shear Fluid Homogenizer (Microfluidic). Next, the lysate was centrifuged at 75,000 $g$ for $30 \mathrm{~min}$, and the supernatant was loaded onto a HisTrap HP $5 \mathrm{ml}$ column (GE Healthcare). The His6 $\times-$ SUMO-NgTAL fusion protein was then eluted with buffer $\mathrm{B}(100 \mathrm{mM}$ Tris/ $\mathrm{HCl} \mathrm{pH} \mathrm{7.5,500} \mathrm{mM} \mathrm{imidazole} \mathrm{and}$ $300 \mathrm{mM} \mathrm{NaCl}$ ) and subsequently dialysed against 1 l buffer A overnight while digesting in situ with $2 \mu \mathrm{ml}^{-1}$ SUMO protease (ULP-1). The cleaved SUMO-tags and non-cleaved protein were subsequently separated from the untagged protein using an additional affinity chromatography step as described above. $\mathrm{NgTAL}$ was then subjected to size-exclusion chromatography using a HiLoad 16/60 Superdex 75 prep grade gel filtration column (GE Healthcare) in buffer C (50 mM glycylglycine $\mathrm{pH}$ 8.0). The oxidized and reduced enzyme forms can be quantitatively separated both on the preparatory as well as analytical scale, as shown in Supplementary Fig. 1. NmTAL was purified using the same protocol.

\section{Steady-state kinetic analysis}

For steady-state kinetic analysis of wild-type $\mathrm{NgTAL}$ and $\mathrm{NmTAL}$ and their variants under oxidizing and reducing conditions, a coupled enzymatic assay ${ }^{41}$ was used that monitors the conversion of donor $D$-fructose 6-phophate and acceptor D-erythrose-4-phophate at $340 \mathrm{~nm}$ as previously described ${ }^{42}$. For measurements under reducing conditions, the protein stock was additionally supplemented with $20 \mathrm{mM}$ dithiothreitol (DTT), resulting in a final concentration of $1 \mathrm{mM}$ DTT in the reaction mix. We established that the activity of the auxiliary enzymes is not affected by the addition of DTT. Initial rates were estimated by linear regression of the absorbance signal over the first $5 \mathrm{~s}$ of the measurements or, in cases in which substrate activation was observed, using

$$
A_{340}(t)=A_{0}-\Delta \mathrm{ss} \cdot t+\frac{\Delta \mathrm{ss}-\Delta_{0}}{k_{\mathrm{obs}}} \cdot\left(1-\exp ^{\left(-k_{\mathrm{obs}} \cdot t\right)}\right)
$$

in which $A_{0}$ denotes the starting absorbance at $340 \mathrm{~nm}, \Delta \mathrm{ss}$ the absorbance change at steady-state (steady-state rate), $\Delta 0$ the absorbance change at $t=0$ (initial rate), and $k_{\text {obs }}$ the first-order rate constant of activation.

The obtained steady-state activities were analysed using the Michaelis-Menten equation.

\section{Circular dichroism}

To analyse the secondary structure content and thermal stability of wild-type $N g$ TAL and NmTAL and their variants, far-UV spectra and thermal unfolding data were collected using circular dichroism spectroscopy (Chirascan, Applied Photophysics). Far-UV spectra were collected in the range of 195-260 nm using a concentration of 0.1 or $0.2 \mathrm{mg} \mathrm{ml}^{-1}$ protein in $100 \mathrm{mM} \mathrm{Na}_{2} \mathrm{HPO}_{4}, \mathrm{pH} 8.0$ with a step size of 1 $\mathrm{nm}$ and at least 20 accumulations for $0.5 \mathrm{~s}$ per wavelength. Unfolding data of $0.2 \mathrm{mg} \mathrm{ml}^{-1} \mathrm{NgTAL}$ were collected at $222 \mathrm{~nm}$ from $20-95^{\circ} \mathrm{C}$ (real sample temperature was determined using a temperature probe) with a ramping speed of $1^{\circ} \mathrm{C} \mathrm{min}^{-1}$. Each temperature data point was collected for $10 \mathrm{~s}$.

\section{Analytical ultracentrifugation}

Sedimentation velocity analysis was carried out in a Beckman Coulter ProteomeLab XL I analytical ultracentrifuge at $50,000 \mathrm{rpm}$ and $10^{\circ} \mathrm{C}$ using an An-50 Ti rotor. Concentration profiles were measured with the absorption scanning optics at $280 \mathrm{~nm}$ using the manufacturer's data acquisition software, ProteomeLab XL-I Version 6.0 (Firmware 5.7). Wild-type $\mathrm{NgTAL}$ or the Lys8Ala or Cys38Ser variants were applied in a concentration range of $11-63 \mu \mathrm{M}$ in a buffer containing $0.1 \mathrm{M}$ Tris $\mathrm{pH} 8.0$ in absence or presence of $1 \mathrm{mM}$ TCEP. Experiments were performed in 3- or 12-mm standard double sector centrepieces filled with $100 \mu \mathrm{l}$ or $400 \mu \mathrm{l}$ sample, respectively. For data analysis, a model for diffusion-deconvoluted differential sedimentation coefficient distributions (continuous $c(s)$ distributions), implemented in the program SEDFIT ${ }^{43}$, was used. Partial specific volumes, buffer density and viscosity were calculated from amino acid and buffer compositions, respectively, by the program SEDNTERP ${ }^{44}$ and were used to correct experimental $s$-values to $s_{20, w}$. Figures were prepared using the program GUSSI ${ }^{45}$.

\section{Mass spectrometry}

Protein sequence information. Sequences for $\mathrm{NgTAL}$ are as follows. For wild type (Lys8 and Cys38 that form the crosslink are in boldface and underlined), G_MTILSDVㅌALGQQIWLDNLSRLVQSGE LAQMLKQGVCGVTSNPAIFQKAFAGDALYADEVAALKRQNLSPKQRYETM AVADVRAACDVCLAEHESTGGKTGFVSLEVSPELAKDAQGTVEEARRLHA AIARKNAMIKVPATDAGIDALETLVSDGISVNLTLLFSRAQTLKAYAAYARG 
IAKRLAAGQSVAHIQVVASFFISRVDSALDATLPDRLKGKTAIALAKAAYQD WEQYFTAPEFAALEAQGANRVQLLWASTGVKNPAYPDTLYVDSLIGVHT VNTVPDATLKAFIDHGTAKATLTESADEARARLAEIAALGIDVETLAARLQE DGLKQFEEAFEKLLAPLV

For the Lys8Ala variant, G_MTILSDVAALGQQIWLDNLSRSLVQSG ELAQMLKQGVCGVTSNPAIFQKAFAGDALYADEVAALKRQNLSPKQRYET MAVADVRAACDVCLAEHESTGGKTGFVSLEVSPELAKDAQGTVEEARRLH AAIARKNAMIKVPATDAGIDALETLVSDGISVNLTLLFSRAQTLKAYAAYAR GIAKRLAAGQSVAHIQVVASFFISRVDSALDATLPDRLKGKTAIALAKAAYQ DWEQYFTAPEFAALEAQGANRVQLLWASTGVKNPAYPDTLYVDSLIGVHTV NTVPDATLKAFIDHGTAKATLTESADEARARLAEIAALGIDVETLAARLQE DGLKQFEEAFEKLLAPLV

For the Cys38Ser variant, G_MTILSDVKALGQQIWLDNLSRSLVQSG ELAQMLKQGVSGVTSNPAIFQKAFAGDALYADEVAALKRQNLSPKQRYET MAVADVRAACDVCLAEHESTGGKTGFVSLEVSPELAKDAQGTVEEARRLH AAIARKNAMIKVPATDAGIDALETLVSDGISVNLTLLFSRAQTLKAYAAYAR GIAKRLAAGQSVAHIQVVASFFISRVDSALDATLPDRLKGKTAIALAKAAY QDWEQYFTAPEFAALEAQGANRVQLLWASTGVKNPAYPDTLYVDSLIGVH TVNTVPDATLKAFIDHGTAKATLTESADEARARLAEIAALGIDVETLAARL QEDGLKQFEEAFEKLLAPLV

\section{Protein digestion}

Proteins from wild-type $\mathrm{NgTAL}$ and the Lys8Ala and Cys38Ser variants with or without DTT ( $1 \mathrm{mM}$ or $5 \mathrm{mM})$ were separately digested by adding trypsin (Promega) with a protein:enzyme ratio of 25:1 (w/w). Trypsin specifically cleaves at the carboxylic side of lysine and arginine residues. The samples were incubated at $37^{\circ} \mathrm{C}$ on a thermomixer overnight. The digested samples were acidified by adding trifluoroacetic acid to a final concentration of $1 \%(v / v)$. The insoluble particles in the samples were removed by centrifuging at $14,000 \mathrm{~g}$ for $10 \mathrm{~min}$ using a benchtop centrifuge. After cleaning up with Oasis HBL columns (Waters), the resulting peptides were dried in a SpeedVac concentrator.

\section{Liquid chromatography-mass spectrometry analysis}

Peptides were resuspended in 5\% (v/v) acetonitrile (ACN), 0.1\% (v/v) formic acid and subjected to liquid chromatography with tandem mass spectrometry analysis using Orbitrap Exploris 480 or Q Exactive (Thermo Scientific), both coupled to a Dionex UltiMate 3000 UHPLC system (Thermo Scientific). Peptides were first concentrated on a $\mathrm{C} 18$ trap column (3-cm long; inner diameter of $100 \mu \mathrm{m}$; outer diameter of $360 \mu \mathrm{m})$ for $3 \mathrm{~min}$, and then separated on a home-made analytical column (ReproSil-Pur 120 C18-AQ, 1.9- $\mu$ m pore size, $75-\mu$ m inner diameter, Dr. Maisch, $30 \mathrm{~cm}$ ) using a 1-h gradient at a flow rate of $300 \mathrm{nl} \mathrm{min}{ }^{-1}$. Mobile phase A was $0.1 \%(\mathrm{v} / \mathrm{v}) \mathrm{FA}$, and mobile phase B was $80 \%(\mathrm{v} / \mathrm{v}) \mathrm{ACN}$ and $0.1 \%(\mathrm{v} / \mathrm{v})$ FA. The gradient started at $10 \% \mathrm{~B}$ at $3 \mathrm{~min}$, increased to $46 \% \mathrm{~B}$ in $42 \mathrm{~min}$. After washing with $90 \% \mathrm{~B}$ for $6 \mathrm{~min}$, the column was re-equilibrated with $5 \% \mathrm{~B}$. The instrument settings were as follows. MS1 settings: detector type, Orbitrap; Orbitrap resolution, 70,000; scan range, $180-1,400 \mathrm{~m} / \mathrm{z}$; maximum injection time, $50 \mathrm{~ms}$; and automatic gain control (AGC) target, $1 \times 10^{6}$. MS2 settings: isolation mode, quadrupole; isolation window, $2 \mathrm{~m} / z$; scan range mode, $200-2,000 \mathrm{~m} / \mathrm{z}$; first mass, $110 \mathrm{~m} / z$; activation type, higher energy collision dissociation; collision energy (\%), 30; detector type, Orbitrap; Orbitrap resolution, 17,500; maximum injection time, $54 \mathrm{~ms}$; AGC target, $1 \times 10^{5}$.

\section{Data analysis}

The raw data were processed using the MaxQuant software package using the following settings: fully specific trypsin digestion, maximum of two missed cleavages, mass tolerance for precursors and fragment ions of 10 and $20 \mathrm{ppm}$, respectively, methionine oxidation as a variable modification, and target false-discovery rate as $1 \%$. For the protein database, the reviewed protein sequences from $N$. gonorrhoeae were downloaded from UniProt. The amino acid sequences of $\mathrm{NgTAL}$ from wild type, and the Lys8Ala and Cys38Ser variants were added to the protein database. Dependent peptide search was activated.

\section{Crystallization and cryoprotection}

Protein crystals of $\mathrm{NgTAL}$ were grown at $20^{\circ} \mathrm{C}$ using the sitting-drop vapour diffusion method with a reservoir solution containing $0.2 \mathrm{M}$ $\mathrm{NaCl}, 20 \%$ PEG $8000,33-40 \mathrm{mM}$ of citric acid and 60-66 mM sodium phosphate. Reservoir solution $(1 \mu \mathrm{l})$ was mixed with protein solution

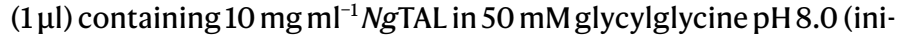
tially supplemented with $1 \mathrm{mMDTT}$ ). In case of acetate conditions, we used a reservoir solution containing $0.2 \mathrm{M} \mathrm{NaCl}, 16 \% \mathrm{PEG} 8000$ and $0.1 \mathrm{M}$ ammonium acetate $\mathrm{pH} 4.2$ and mixed $2 \mu$ l protein solution containing $4 \mathrm{mg} \mathrm{ml}^{-1}$ wild-type $\mathrm{NgTAL}$ in $50 \mathrm{mM}$ glycylglycine $\mathrm{pH} 8.0$ with $2 \mu \mathrm{l}$ reservoir solution. For crystallization of the reduced protein, we mixed $2 \mu \mathrm{l}$ reservoir solution composed of $0.2 \mathrm{M} \mathrm{NaCl}, 16 \%$ PEG 8000, $0.1 \mathrm{M}$ citric acid $\mathrm{pH} 4.2$ and $5 \%(\mathrm{v} / \mathrm{v}) \beta$-mercaptoethanol with $2 \mu$ l protein solution containing $10 \mathrm{mg} \mathrm{ml}^{-1}$ wild-type $\mathrm{NgTAL}$ in $50 \mathrm{mM}$ glycylglycine $\mathrm{pH}$ 8.0. Cryoprotection was performed with $20 \%(\mathrm{w} / \mathrm{v})$ glycerol in well solution, soaking the crystals for up to $90 \mathrm{~s}$.

\section{X-ray data collection, processing and model building}

Diffraction data of single NgTAL crystals were collected using synchrotron radiation at the beamline P14 of DESY EMBL at a wavelength of $0.73 \AA$ at $100 \mathrm{~K}$ with an EIGER $16 \mathrm{M}$ detector or, alternatively, at an in-house rotating anode (Rigaku MicroMax-003) equipped with a Dectris Pilatus $200 \mathrm{~K}$ detector. Processing was performed using the XDS package ${ }^{46}$. Subsequent refinement and model building was performed employing Phenix.refine ${ }^{47}$ and $\operatorname{Coot}^{48}$. Phasing was performed by rigid-body refinement using the deposited NgTAL structure (PDB code 3CLM) as the initial model. The geometry of the structure was validated using MolProbity ${ }^{49}$. Representations of structures were prepared using $\mathrm{PyMOL}^{50}$. Omit density maps were calculated using Phenix.polder ${ }^{51}$. The Ramachandran statistics are $99.14 \%$ in the favoured and $0.86 \%$ in the allowed region for $6 Z \mathrm{ZX}, 98.27 \%$ and $1.73 \%$ for $6 \mathrm{ZWJ}, 98.86 \%$ and $1.14 \%$ for $6 Z \mathrm{WF}, 98.85 \%$ and $1.15 \%$ for $7 \mathrm{BOL}, 98.86 \%$ and $1.14 \%$ for $7 \mathrm{BBX}$ and $98.42 \%$ and $1.58 \%$ for $7 \mathrm{BBW}$.

The search for potential NOS bridges in protein structures deposited in the PDB was carried out as previously detailed ${ }^{18}$.

\section{Computational details}

All calculations were carried out with the Gaussian16 program package $^{52}$, making use of the B3LYP functional ${ }^{53}$ and a previously published D3 empirical dispersion correction (Becke-Johnson damping applied throughout $)^{54}$. Geometry and frequency calculations were carried out using the def2-SVPD basis set ${ }^{55}$. Free energy corrections were computed from the harmonic frequencies under standard state conditions ( $T=298.15 \mathrm{~K})$. The electronic energy was refined at the B3LYP-D3(BJ)/ def2-TZVP level of theory. All calculations were performed with the SMD solvation model using the permitivity of water and default settings. Different reaction intermediates were computed using a reduced cluster model around the NOS linkage we studied. The latter consisted of Cys38, Glu93, Thr101 and Lys8, all capped at the $\alpha$-carbon atom, and with the Cartesian coordinates of the latter constrained to their crystallographic positions. Three water molecules found in the crystal structure were also included. For each intermediate, three protonation states were optimized (total system charge of $-1,0$ and 1 ), $\mathrm{p} K_{\mathrm{a}}$ values were computed and the most stable species was considered (taking $\mathrm{pH}=8$, the value at which activity was measured). The energies for selected intermediates are provided in Extended Data Fig. 5.

\section{Reporting summary}

Further information on research design is available in the Nature Research Reporting Summary linked to this paper.

\section{Data availability}

The refined structural protein models and corresponding structure-factor amplitudes have been deposited under PDB accession 
codes 6XZ4 (NgTAL oxidized citrate 1), 6ZWJ (NgTAL oxidized citrate 2), 6ZWH (NgTAL oxidized acetate), 6ZWF (NgTAL reduced citrate), 7BOL (NgTAL oxidized low-dose), 7BBX (NgTAL Lys8Ala variant) and 7BBW (NgTAL Cys38Ser variant). The structures cited in this publication (1M3Q, 3CLM, 6T3X and 5Y72) are available under their respective $\mathrm{PDB}$ accession codes. The data for our protein database are currently private, and can only be accessed with a single reviewer account that has been created. The mass spectrometry proteomics data have been deposited to the ProteomeXchange Consortium via the PRIDE partner repository with the dataset identifier PXD020302 (reviewer account details: reviewer52532@ebi.ac.uk (username),AK3E73R2 (password)). All other data are available from the corresponding author on request.

38. Gill, S. C. \& von Hippel, P. H. Calculation of protein extinction coefficients from amino acid sequence data. Anal. Biochem. 182, 319-326 (1989).

39. Inoue, H., Nojima, H. \& Okayama, H. High efficiency transformation of Escherichia coli with plasmids. Gene 96, 23-28 (1990)

40. Bertani, G. Studies on lysogenesis. I. The mode of phage liberation by lysogenic Escherichia coli. J. Bacteriol. 62, 293-300 (1951).

41. Tsolas, O. \& Joris, L. Transaldolase. Methods Enzymol. 42, 290-297 (1975).

42. Sautner, V., Friedrich, M. M., Lehwess-Litzmann, A. \& Tittmann, K. Converting transaldolase into aldolase through swapping of the multifunctional acid-base catalyst: common and divergent catalytic principles in F6P aldolase and transaldolase. Biochemistry 54, 4475-4486 (2015).

43. Schuck, P. Size-distribution analysis of macromolecules by sedimentation velocity ultracentrifugation and lamm equation modeling. Biophys. J. 78, 1606-1619 (2000).

44. Laue, M. T., Shah, B. D., Rigdeway, T. M. \& Pelletier, S. L. in Analytical Ultracentrifugation in Biochemistry and Polymer Science (eds Harding S. et al.) 90-125 (Royal Society of Chemistry, 1992).

45. Brautigam, C. A. Calculations and publication-quality illustrations for analytical ultracentrifugation data. Methods Enzymol. 562, 109-133 (2015).

46. Kabsch, W. Xds. Acta Crystallogr. D 66, 125-132 (2010).

47. Adams, P. D. et al. PHENIX: a comprehensive Python-based system for macromolecular structure solution. Acta Crystallogr. D 66, 213-221 (2010).

48. Emsley, P., Lohkamp, B., Scott, W. G. \& Cowtan, K. Features and development of Coot. Acta Crystallogr. D 66, 486-501 (2010).

49. Williams, C. J. et al. MolProbity: More and better reference data for improved all-atom structure validation. Protein Sci. 27, 293-315 (2018).

50. Schrödinger. The PyMOL Molecular Graphics System, version 1.8 (2015).

51. Liebschner, D. et al. Polder maps: improving OMIT maps by excluding bulk solvent. Acta Crystallogr. D 73, 148-157 (2017).
52. Frisch, M. J. et al. Gaussian 16, revision C.01 (Gaussian, 2016.

53. Becke, A. D. Density-functional thermochemistry. III. The role of exact exchange. J. Chem. Phys. 98, 5648-5652 (1993).

54. Grimme, S., Ehrlich, S. \& Goerigk, L. Effect of the damping function in dispersion corrected density functional theory. J. Comput. Chem. 32, 1456-1465 (2011).

55. Rappoport, D. \& Furche, F. Property-optimized Gaussian basis sets for molecular response calculations. J. Chem. Phys. 133, 134105 (2010).

56. Wong, C. P. et al. Two distinct substrate binding modes for the normal and reverse prenylation of hapalindoles by the prenyltransferase AmbP3. Angew. Chem. Int. Ed. 57, 560-563 (2018).

57. Muller, Y. A. et al. High-resolution crystal structures of two prototypical $\beta$ - and $y$-herpesviral nuclear egress complexes unravel the determinants of subfamily specificity. J. Biol. Chem. 295, 3189-3201 (2020).

Acknowledgements This study was supported by the Max-Planck Society and the DFG-funded Göttingen Graduate Center for Neurosciences, Biophysics and Molecular Biosciences GGNB. We acknowledge access to beamline P14 at DESY/EMBL (Hamburg), and thank G. Bourenkov and T. Schneider for local support. We thank G. Bourenkov, A. Pearson, H. Stark, R. Kluger and M. Alcarazo for discussion; and A. Linden for help with the mass spectrometry experiments.

Author contributions K.T. designed and coordinated research; M.W. expressed, purified, biochemically characterized and crystallized NgTAL proteins with initial support from P.K.; L.-M.F. expressed, purified and biochemically characterized NmTAL; F.R.v.P. and V.S. conducted crystallographic data collection, processing, model building and refinement with support from K.T.; F.R.v.P., V.S. and K.T. interpreted crystallographic data; F.R.v.P. did phylogenetic analysis; U.C. conducted and analysed analytical ultracentrifugation experiments; P.F. and K.-T.P. performed and analysed mass spectrometry experiments under the supervision of H.U.; R.A.M. supervised and planned theoretical investigations; J.U. performed electronic structure calculations including potentials, $\mathrm{p} K_{\mathrm{a}}$ value and structures of reaction intermediates; J.Y. performed electronic structure calculations of reaction intermediates and pathways; U.D., J.U., R.A.M. and K.T. discussed reaction mechanisms; K.T. wrote the manuscript with input from all authors.

Competing interests M.W., F.R.v.P., L.-M.F., U.D., J.U., R.A.M., V.S. and K.T. have filed a European patent application (application number EP21164101.4) for regulating protein activities by targeting the NOS redox switches.

Additional information

Supplementary information The online version contains supplementary material available at https://doi.org/10.1038/s41586-021-03513-3.

Correspondence and requests for materials should be addressed to K.T.

Peer review information Nature thanks Deborah Fass, Martin Högbom and Judith P. Klinman

for their contribution to the peer review of this work. Peer reviewer reports are available. Reprints and permissions information is available at http://www.nature.com/reprints. 
a

a $\mathrm{C}_{\mathrm{s}}^{\mathrm{s}}$ disulfide (intramolecular)

thiol ${ }_{\text {sulfenic acid }}^{\stackrel{\mathrm{ROS}}{\mathrm{RSH}}}$

pathophysiology

b

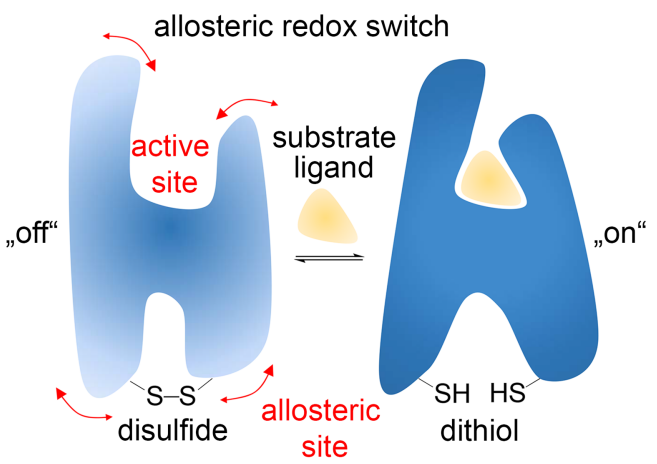

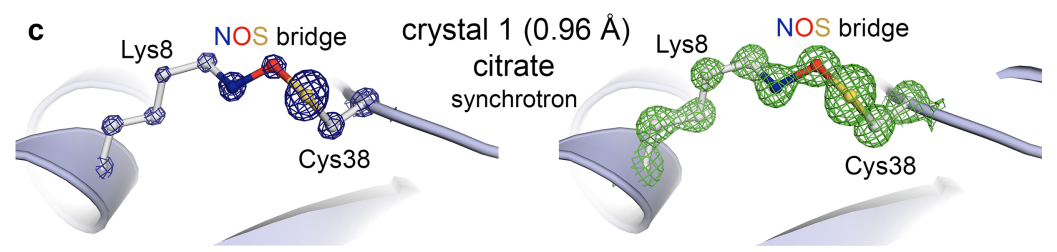
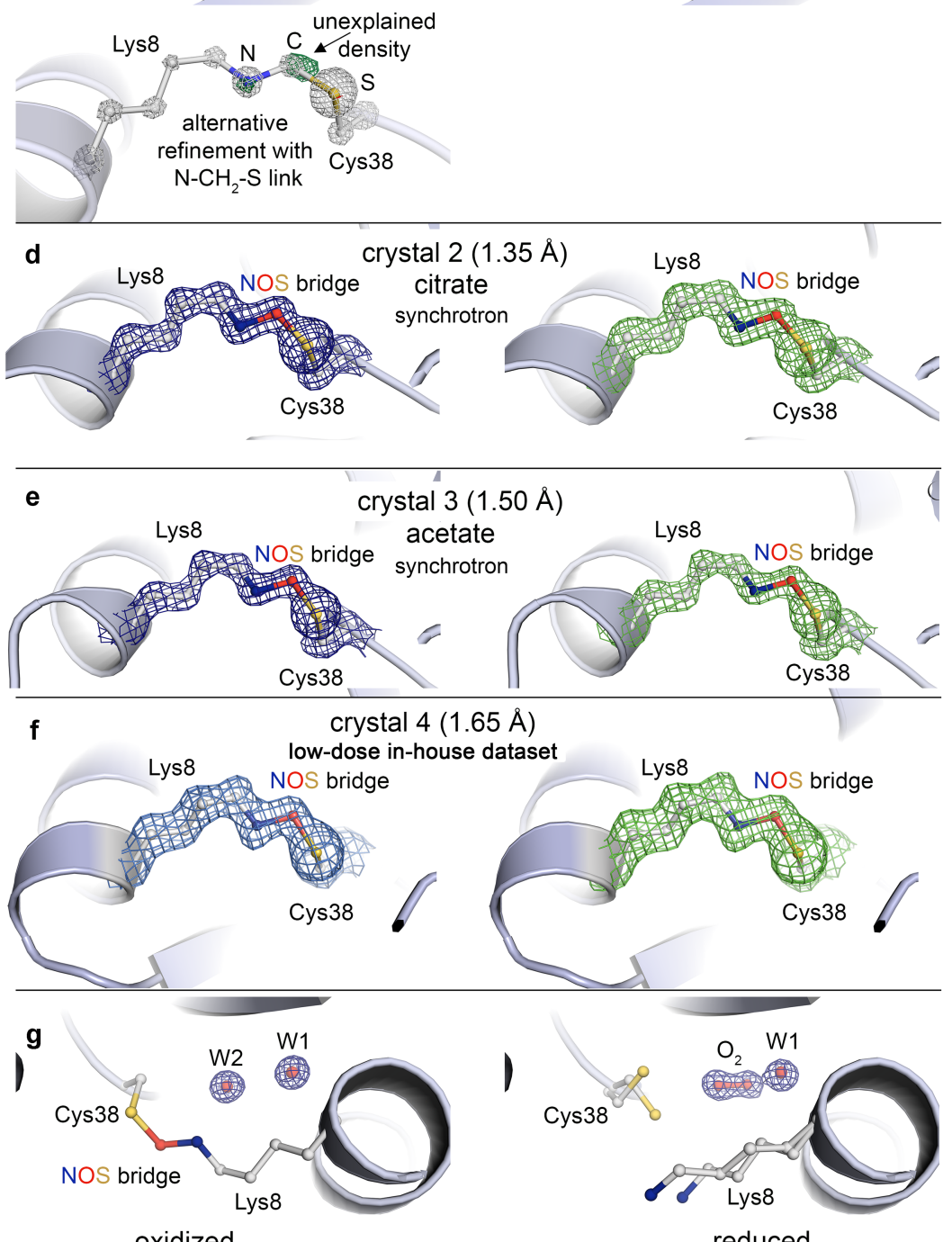

oxidized

reduced

Extended Data Fig. 1 | See next page for caption. 
Extended Data Fig. $1 \mid$ Redox chemistry of cysteine residues in proteins and electron density maps, showing the presence of the lysine-cysteine NOS bridge in independent crystal structures of $\mathrm{NgTAL}$. a, Redox reactions of cysteine with key species involved, including cysteine oxidation by reactive oxygen species (ROS) and reduction by thiol antioxidants (RSH) or enzymes (Srx).b, Mechanism of allosteric redox switch containing an intramolecular allosteric disulfide bridge. Reduction of the disulfide to the corresponding dithiol results in a structural reorganization of the protein that is propagated to the active-site altering activity. c, $N g$ TAL crystal 1 . Top left, $2 m F_{\mathrm{o}}-D F_{\mathrm{c}}$ electron density map after final refinement contoured at $3 \sigma$. No additional positive or negative electron density was observed at $\pm 3 \sigma$. Top right, $m F_{\mathrm{o}}-D F_{\mathrm{c}}$ omit electron density map contoured at $5 \sigma$. Bottom, alternative refinement with a methylene bridge yields unexplained positive difference electron density at the bridging atom. The $2 m F_{\mathrm{o}}-D F_{\mathrm{c}}$ map is shown in grey at $3 \sigma$, positive (green) and negative (red) electron density maps are shown at $3 \sigma$ and $-3 \sigma$, respectively. d, $N g$ TAL crystal 2. Left, $2 m F_{\mathrm{o}}-D F_{\mathrm{c}}$ electron density map after final refinement, contoured at $1.5 \sigma$. Right, $m F_{\mathrm{o}}-D F_{\mathrm{c}}$ omit electron density map contoured at $5 \sigma$. e, $N g$ TAL crystal 3. Left, $2 m F_{\mathrm{o}}-D F_{\mathrm{c}}$ electron density map after final refinement, contoured at 1.5o. Right, $m F_{\mathrm{o}}-D F_{\mathrm{c}}$ omit electron density map contoured at $5 \sigma . \mathbf{f}, \mathrm{NgTAL}$ crystal 4 . Low-dose dataset of an independent crystal measured in-house at a rotating anode. The NOS bridge is also present in the corresponding $N g T A L$ structure (left, $2 m F_{\mathrm{o}}-D F_{\mathrm{c}}$ electron density map contoured at $1 \sigma$; right, $m F_{\mathrm{o}}-D F_{\mathrm{c}}$ omit electron density map contoured at $3 \sigma$ ), thus ruling out that formation of the covalent linkage seen in crystals 1-3 results from radiation damage at the high-energy synchrotron beamline. g, Structure of the Lys8-Cys38 redox switch in the oxidized (left) and reduced (right) state, showing electron density for neighbouring waters (W1 and W2) and a presumed dioxygen molecule $\left(\mathrm{O}_{2}\right)$ that is exclusively observed in the reduced state. The corresponding $2 m F_{\mathrm{o}}-D F_{\mathrm{c}}$ electron density map is shown in blue at a contour level of $1.5 \sigma$. 


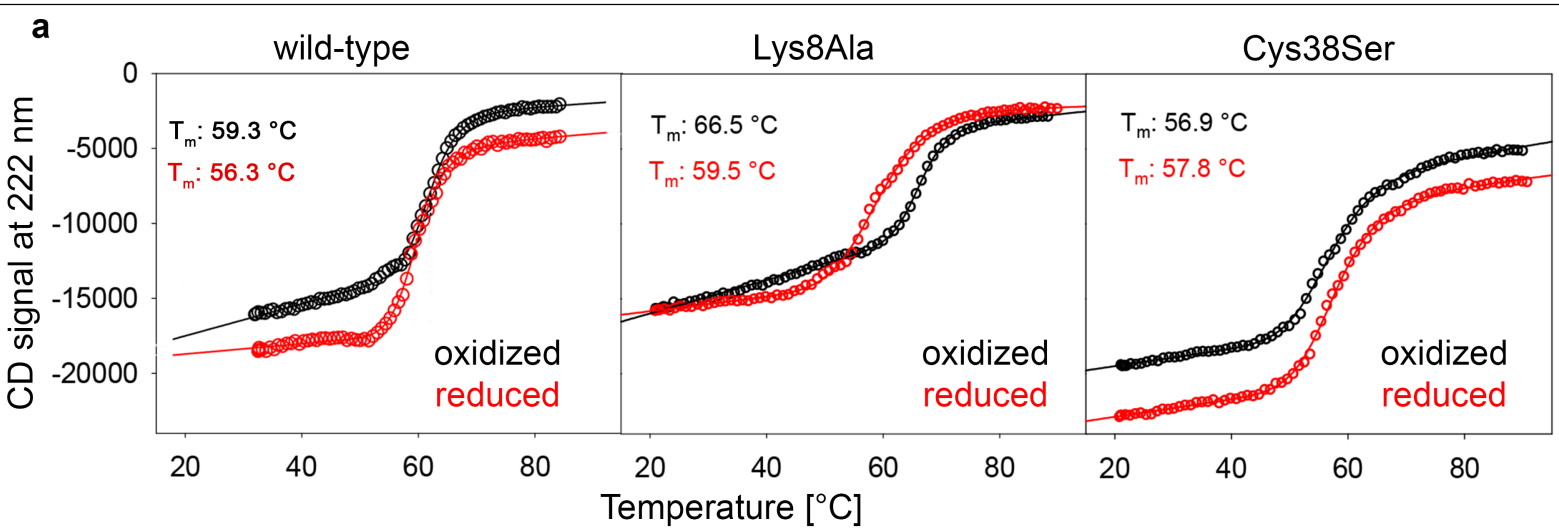

b
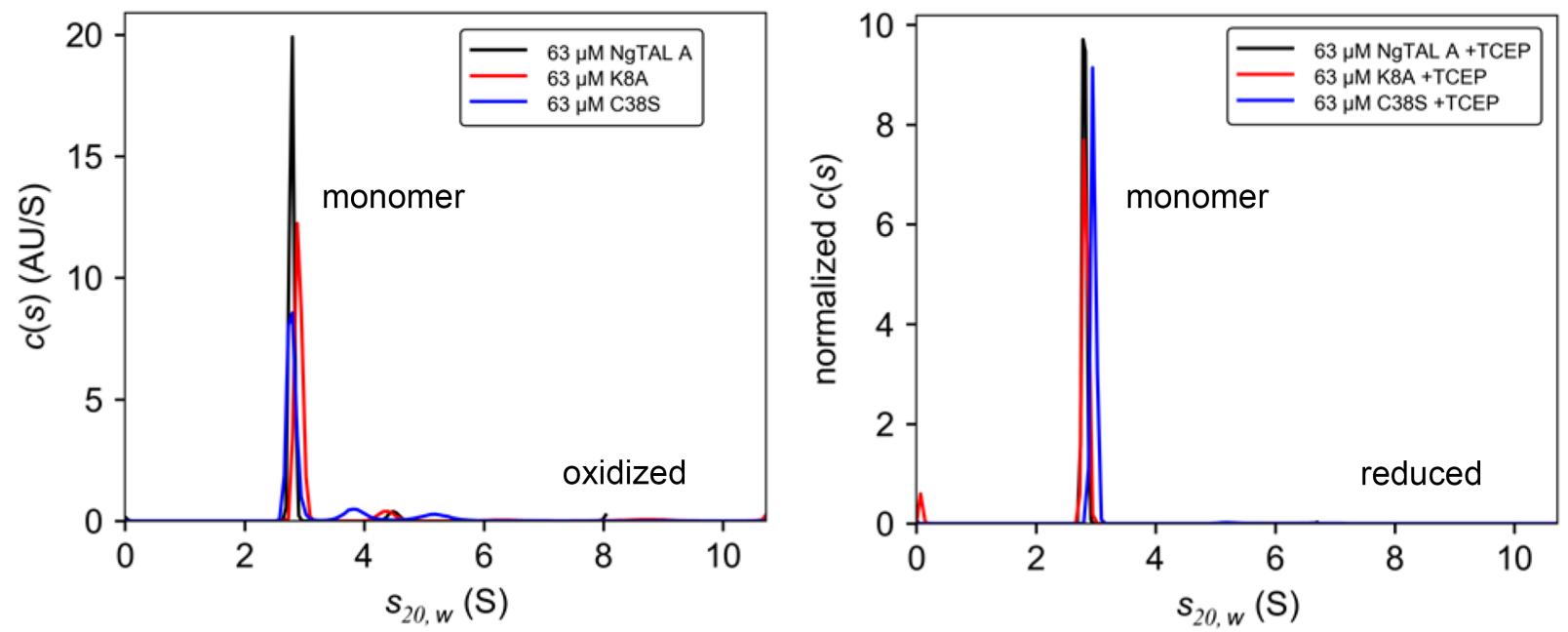

c

NgTAL Lys8Ala $(0.85 \AA)$

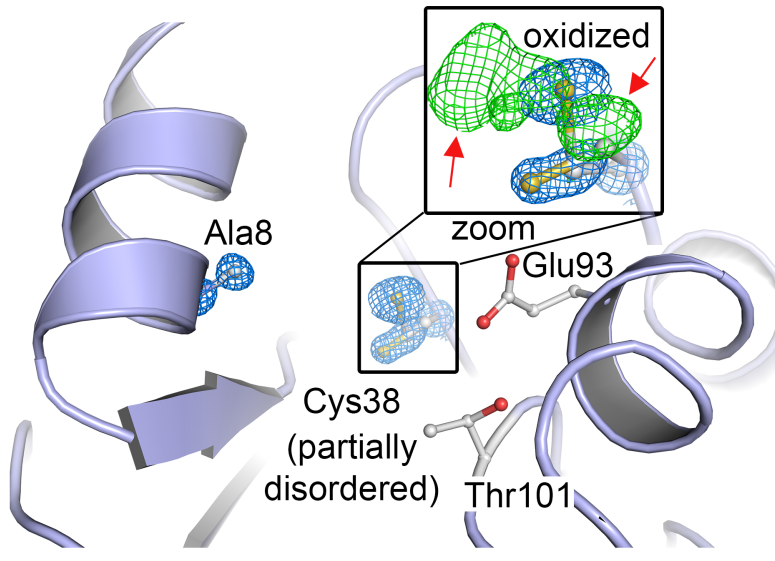

d $\quad \operatorname{NgTAL}$ Cys38Ser $(1.25 \AA)$

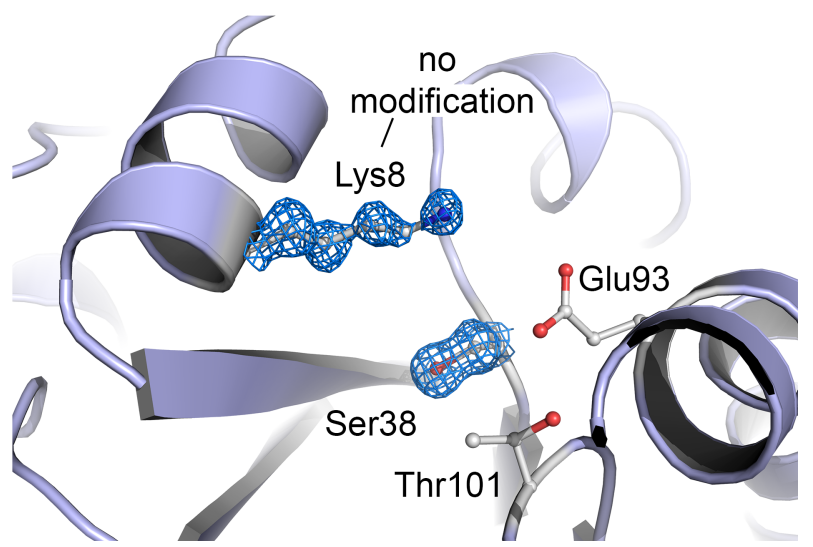

Extended Data Fig. 2 |See next page for caption. 
Extended Data Fig. 2 | Thermal unfolding, analytical ultracentrifugation and X-ray structures of $\mathrm{NgTAL}$ Lys8Ala and Cys38Ser variants. a, Thermal unfolding of $\mathrm{NgTAL}$ wild type and the Lys8Ala and Cys38Ser variants under oxidizing and reducing conditions, as monitored by far-UV CD spectroscopy at $222 \mathrm{~nm}$. Different unfolding temperatures are seen for the oxidized and reduced states in case of the wild-type enzyme and the Lys8Ala variant, whereas the Cys38Ser variant does not exhibit this feature. This suggests an oxidation of Cys 38 in the Lys8Ala variant, despite the absence of Lys8. b, Analytical ultracentrifugation analyses of $\mathrm{NgTAL}$ wild-type and variants in the oxidized and reduced state shows the predominant formation of the monomeric form in all of the cases we tested. Under oxidizing conditions and high protein concentrations, a small fraction of higher oligomers is observed (presumably resulting from incorrectly linked monomers). c, X-ray crystallographic structure of the $N g$ TAL Lys8Ala variant, showing the allosteric redox switch site with residues Ala8 (mutation site), Cys38, Glu93 and Thr101.
For residues Ala8 and Cys 38 , the corresponding $2 m F_{o}-D F_{c}$ electron density maps are shown in blue at a contour level of $2 \sigma$. Inset, peaks in the $m F_{\mathrm{o}}-D F_{\mathrm{c}}$ difference electron density map (in green, contour level $3 \sigma$ ) around the sulfur atom of residue Cys 38 suggest that this atom is oxidized. Owing to the structural flexibility of Cys38, the discrete oxidation state (mono-oxidized and/or dioxidized) cannot be unambiguously assigned. Notwithstanding this ambiguity, this observation supports our proposed mechanism of an initial cysteine oxidation as part of the formation of the NOS bridge. $d, X$-ray crystallographic structure of the $\mathrm{NgTAL}$ Cys38Ser variant, showing the allosteric redox switch site with residues Lys8, Ser38 (mutation site), Glu93 and Thr101. For residues Lys 8 and Ser 38 , the corresponding $2 m F_{\mathrm{o}}-D F_{\mathrm{c}}$ electron density maps are shown in blue at a contour level of $1.5 \sigma$. Lys 8 is chemically unmodified, thus ruling out that the covalent linkage between Lys 8 and Cys 38 seen in the wild-type enzyme results from the addition of $\mathrm{CO}_{2}$ or formaldehyde potentially establishing an NCS linkage ${ }^{18}$. 
a $\mathrm{Lys}_{8}-\mathrm{NH}_{3}^{\oplus}$

b

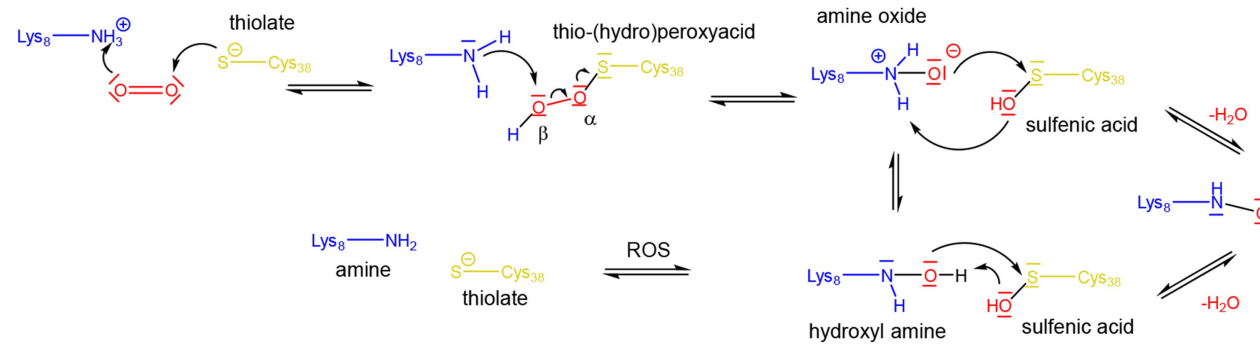

C

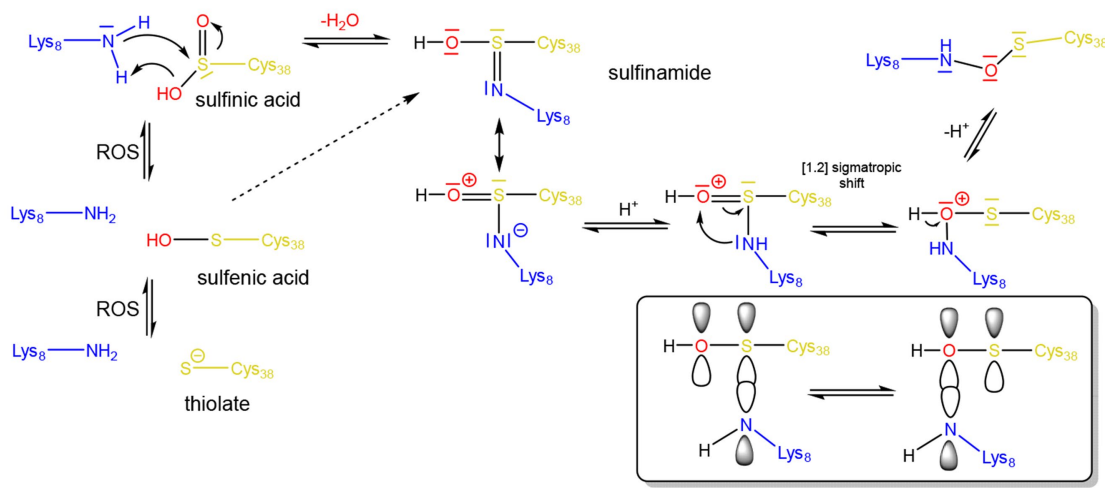

d

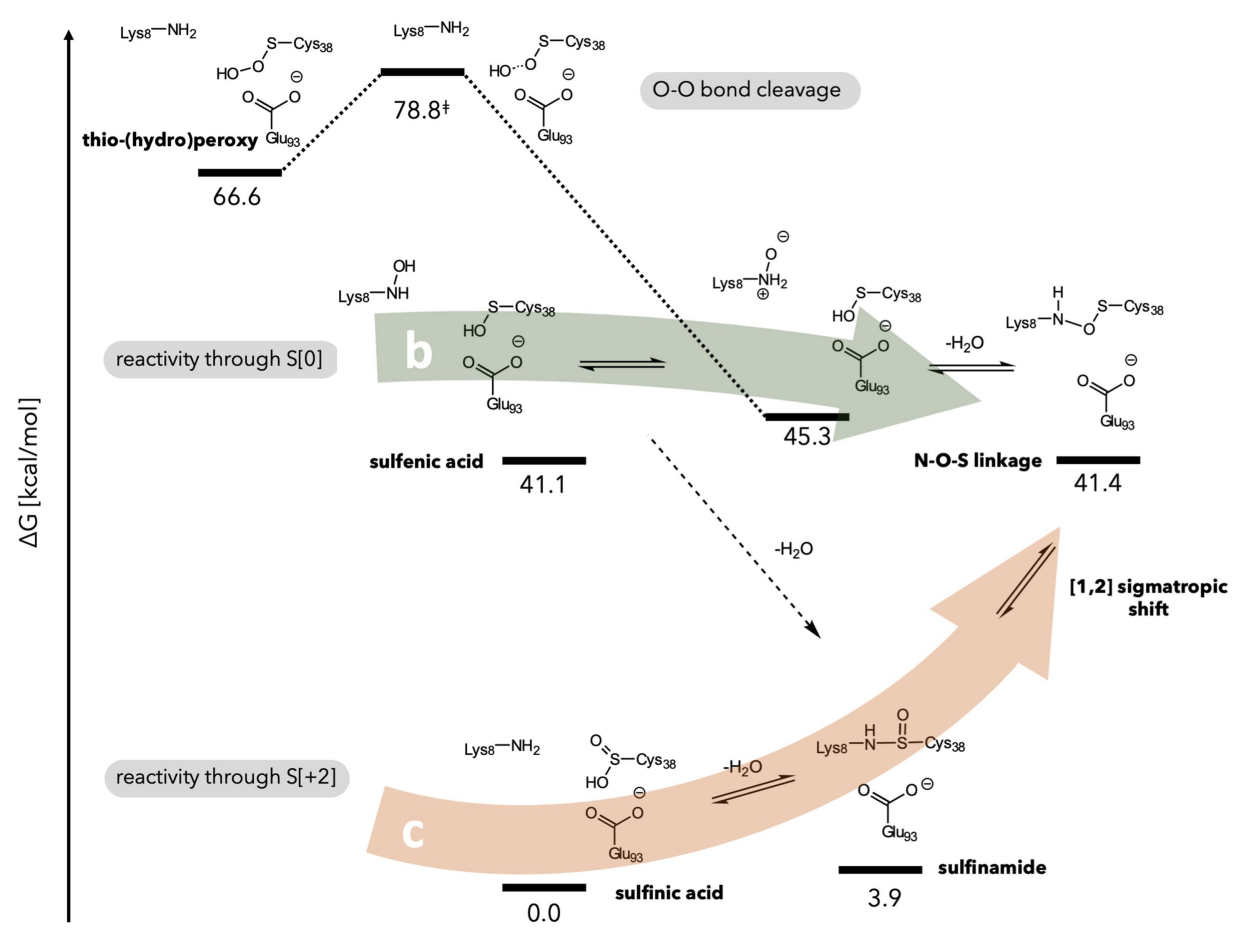

Extended Data Fig. 3 | See next page for caption. 
Extended Data Fig. 3 | Putative reaction mechanisms of lysine-cysteine NOS bridge formation and associated computational calculations. a, Initial reaction of the cysteine thiolate with dioxygen and subsequent attack of the lysine amine onto the $\alpha$-oxygen atom of the thio-(hydro)peroxy intermediate concomitantly with proton transfers and water release. b, Oxidation of both cysteine and lysine in either concerted fashion (top path) or independently (bottom path), followed by nucleophilic attack of the oxidized lysine as a $\mathrm{O}$-nucleophile onto the cysteine sulfenic acid with concomitant water release. c, Initial attack of the lysine amine onto the sulfur atom of sulfenic acid or sulfinic acid to afford a sulfinamide species followed by [1,2] rearrangement driven by orbital steering. d, Depiction of selected reaction intermediates and relative free Gibbs energies $(T=298.15 \mathrm{~K}$ ) computed at the B3LYP-D3(BJ)/def2TZVPD//B3LYP-D3(BJ)/def2-SVPD level of theory (as described in 'Computational details' in Methods). The reference is given by the sulfinic acid state (the thermodynamically most stable intermediate in our investigations). Top, starting thio-(hydro)peroxy species, which bears a barrier of $12.2 \mathrm{kcal}$ $\mathrm{mol}^{-1}$ for the heterolytic cleavage of the $\mathrm{O}-\mathrm{O}$ bond and concurrent oxidation of the lysine residue. We compare two different pathways (using the same nomenclature as in $\mathbf{a}-\mathbf{c}$ ) through the sulfenic and sulfinic acids. The mechanism in a is not directly depicted, as we have not observed any concerted $\mathrm{O}-\mathrm{O}$ cleavage with amino nucleophilic attack and NOS bridge formation. Only the mechanisms in b, $\mathbf{c}$ are depicted. A path that leads through the sulfinic acid or sulfinamide species is not viable, because the two species are far too stable. Instead, we suggest that the formal oxidation state of sulfur [0] is kept, with concurrent oxidation of the Lys8 (pathway from $\mathbf{b}$ ). This would represent a reversible mechanism. For each intermediate, three different protonation states were investigated with a total cluster charge of $-1,0$ and 1 . Only the most stable species are shown. 
24792 Sequences, clades with $B R L<0.5$ collapsed

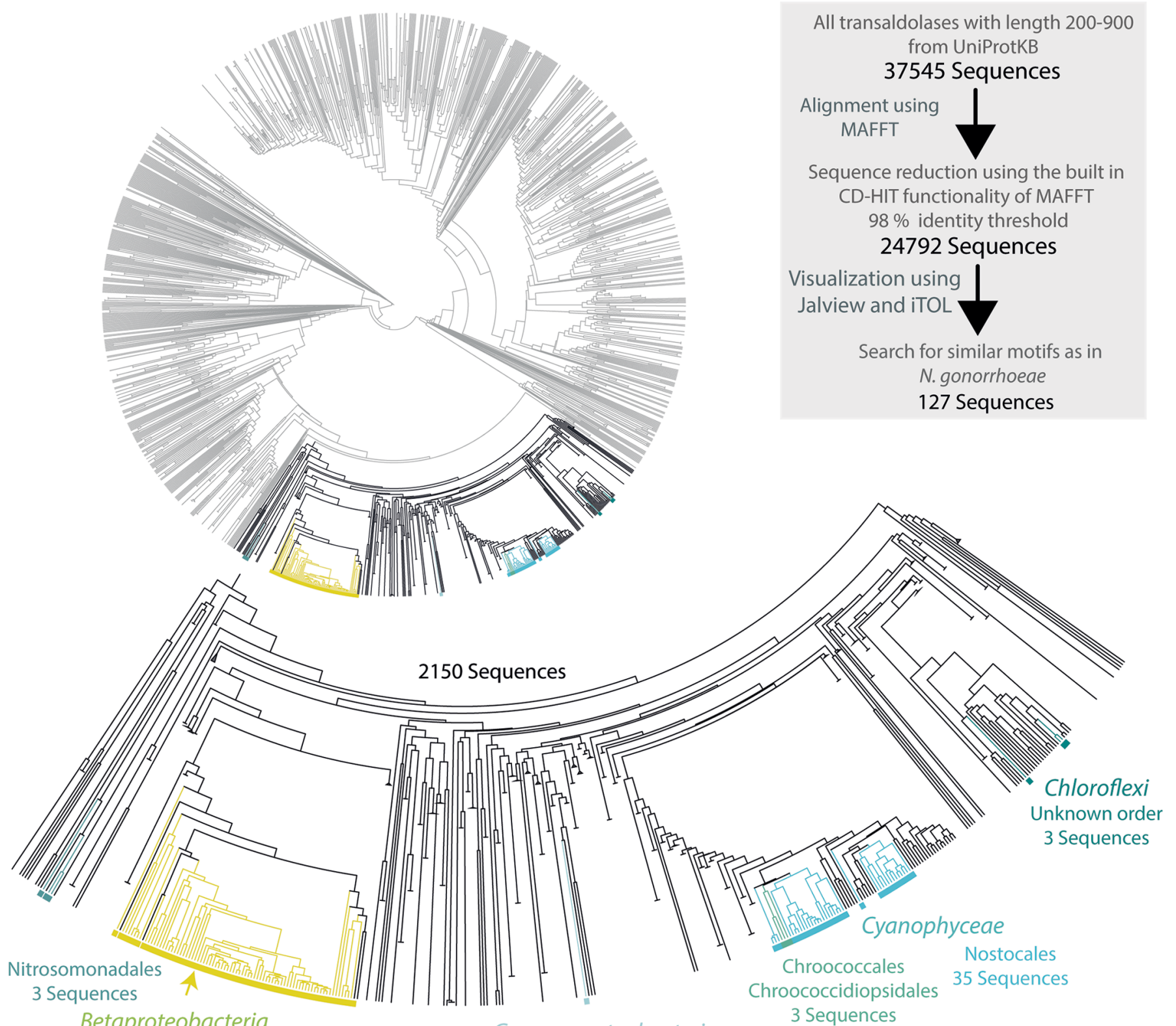

Betaproteobacteria

Neisseriales

76 Sequences
Gammaproteobacteria

Unknown order

1 Sequence

8

\begin{abstract}
N. gonorrhoeae N. meningitidis Motif 1 - Consensus Motif 2 - Consensus Motif consensus
\end{abstract}

MTILSDVKALGQQIWLDNLSRSLVQSGELAQMLK-QGVCGVTSNPAIF MT I LSDVKALGQQ IWLDNLSRS LVQSGELAQMLK-QGVCGVTSNPA I F MT I LSDVKALGQQ IWLDNLSRSLVQSGELAPMLP-QGVCGVTSNPAIF TNPLLE IKPYGQS IWMDNLPRDI I SSGELKPLVENPGICGITSNPS IF -p-L-ahK--GQp I WhDNLpRphhpSGEL-phhp-pGhCGhTSNPS I F redox switch redox switch | active site

Extended Data Fig. 4 | Phylogenetic analyses and sequence conservation of the lysine-cysteine redox switch in the TAL protein family using $\mathrm{NgTAL}$ as reference. Two related consensus motifs were identified that contain the lysine and cysteine residues of the redox switch, the active site serine and asparagine residues required for catalytic activation of the Schiff-base-forming lysine, and the linker region that connects the redox switch with the active site.
The identified motifs are highly conserved in Betaproteobacteria (in particular, Neisseriales (motif 1), and in Cyanophycea (motif 2)). NmTAL is highly similar (95\% identity) to $N g$ TAL, and also contains motif 1 . Structural and functional analyses of NmTAL could confirm the existence of a redox switch (Extended Data Fig. 5). 


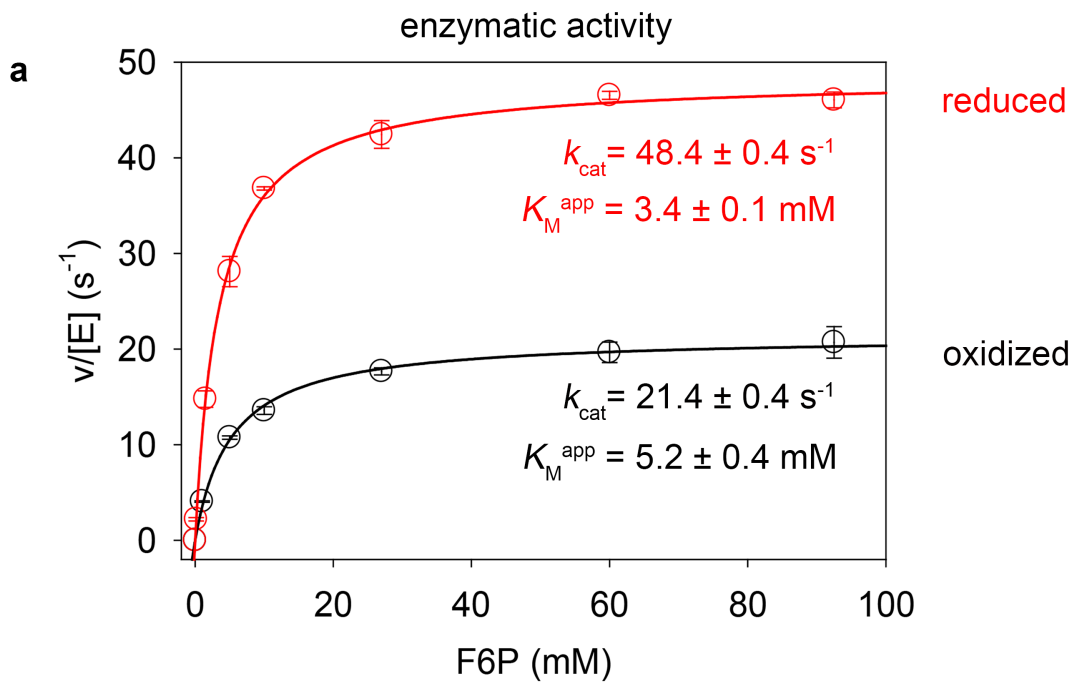

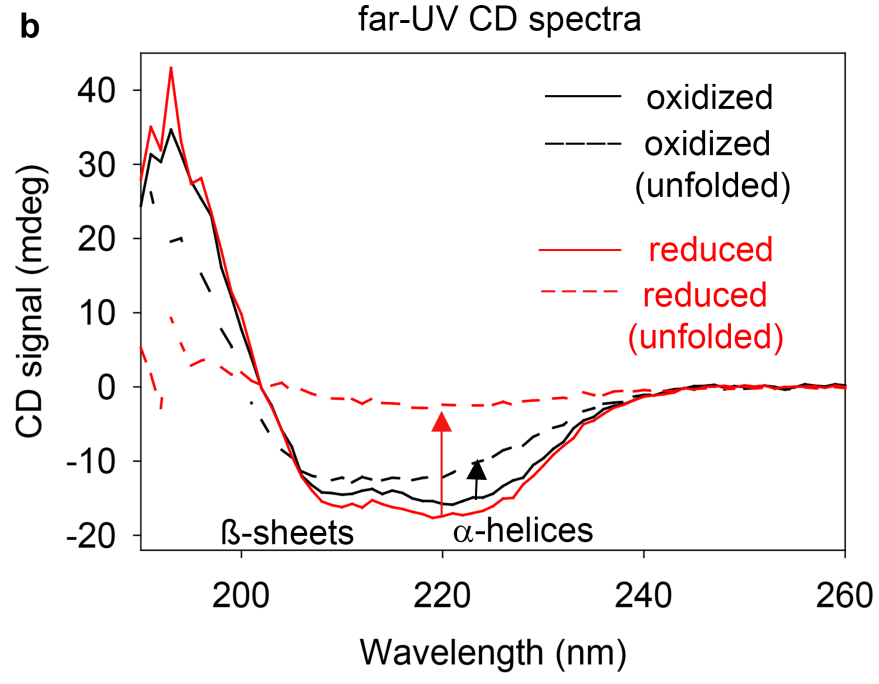

Extended Data Fig. 5 | Structural and functional analyses of $\mathrm{NmTAL}$ indicate the presence of a redox switch. a, Steady-state kinetic analysis of enzymatic activity of NmTAL in the oxidized (black) and reduced (red) state. There is a multi-fold increase of $k_{\text {cat }}$ and concomitant decrease of substrate $K_{\mathrm{M}}$ upon reduction. The catalytic constant of the oxidized form represents an upper limit, as oxidized and reduced species cannot be quantitatively separated by chromatographic methods as in the case of $\mathrm{NgTAL}$. All measurements were carried out in triplicate and are shown as mean \pm s.d. Kinetic and thermodynamic constants are provided in Extended Data Table 1. Experiments were repeated twice with similar results. b, Far-UV CD spectra of NmTAL in the oxidized (black) and reduced (red) state, showing both the natively folded states (solid lines) and states after thermal unfolding (dashed lines). The

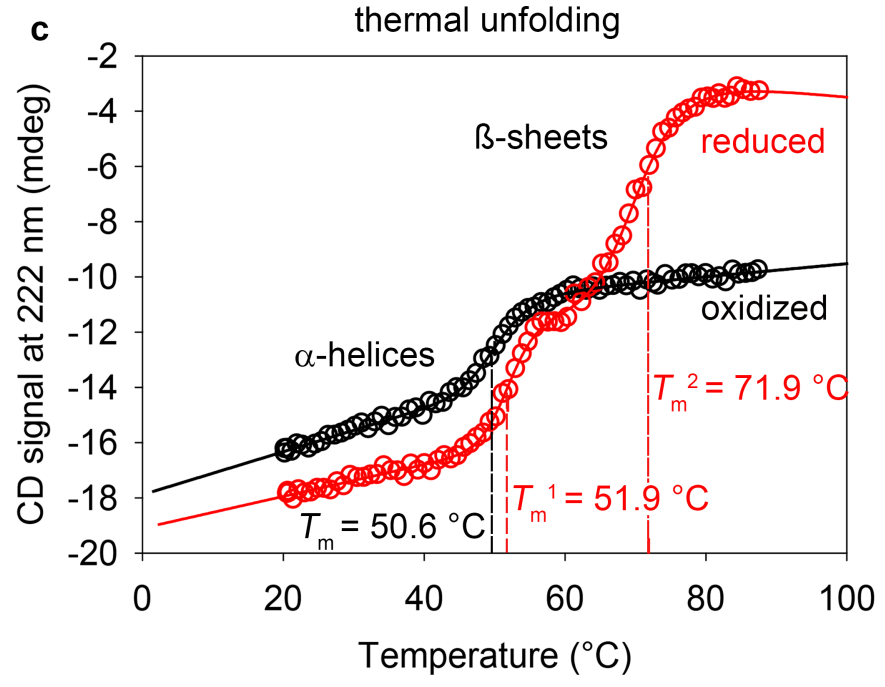

reduced enzyme completely unfolds and does not contain residual secondary structure. By contrast, the oxidized enzyme exhibits only partial unfolding of mostly helical elements and contains thermally stable $\beta$-sheet structures. c, Thermal unfolding of NmTAL in the oxidized (black) and reduced (red) state, monitored by far-UVCD spectroscopy at $222 \mathrm{~nm}$. Although the oxidized enzyme displays a monophasic unfolding with a melting temperature of $50.6^{\circ} \mathrm{C}$ corresponding to unfolding of the $\alpha$-helices (as shown in $\mathbf{b}$ ), the reduced enzyme shows a biphasic unfolding with melting temperatures of $51.9^{\circ} \mathrm{C}$ (first transition) and $71.9^{\circ} \mathrm{C}$ (second transition). This observation suggests that the putative NOS bridge in the oxidized state of NmTAL specifically stabilizes the interior $\beta$-sheet structure of the TIM barrel, in agreement with the position of switch residue Cys38. 


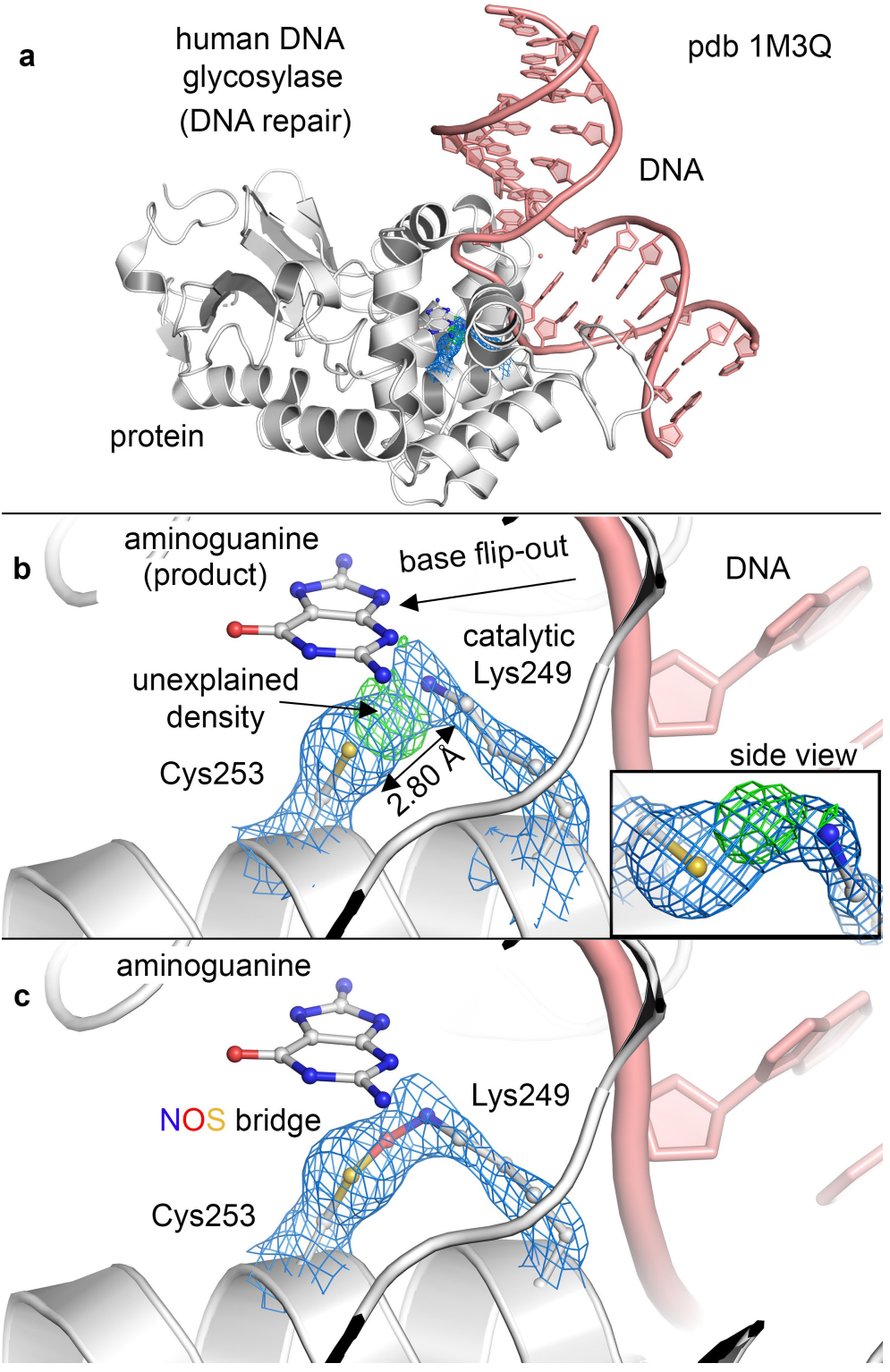

Extended Data Fig. 6 | Structure of the human DNA repair enzyme 8-oxoguanine glycosylase 1 with a putative NOS bridge at the active site. Structure is from PDB $1 \mathrm{M} 3 \mathrm{Q}$, and is at 1.90 A resolution. a, Overall structure of human 8-oxoguanine glucosylase 1 in complex with DNA and product analogue 8 -aminoguanine. b. Close-up view of the active site, showing the product and residues Cys253 and Lys249. Residues Cys253 and Lys249 are superposed with the calculated $2 m F-D F_{\mathrm{c}}$ electron density map (in blue, contour level $1.5 \sigma$ ). A strong, unexplained positive peak in the $m F_{\mathrm{o}}-D F_{\mathrm{c}}$ difference electron density map (in green, contour level $3 \sigma$ ) is observed between the cysteine sulfur atom and the lysine nitrogen atom. The S-N interatomic distance $(2.80 \AA$ in the previously deposited structure) is too short for a hydrogen-bond interaction and too long for a direct S-N linkage.c, Refinement of the previously deposited structure with a geometrically parametrized NOS bridge linking Cys 253 and Lys 249 resulted in a structural model with no remaining unexplained electron density $\left(2 m F_{\mathrm{o}}-D F_{\mathrm{c}}\right.$ electron density map in blue, contour level $1.5 \sigma ; m F_{\mathrm{o}}-D F_{\mathrm{c}}$ difference electron density map in green and red, contour level $\pm 3 \sigma$ ), suggesting the presence of an NOS bridge. 


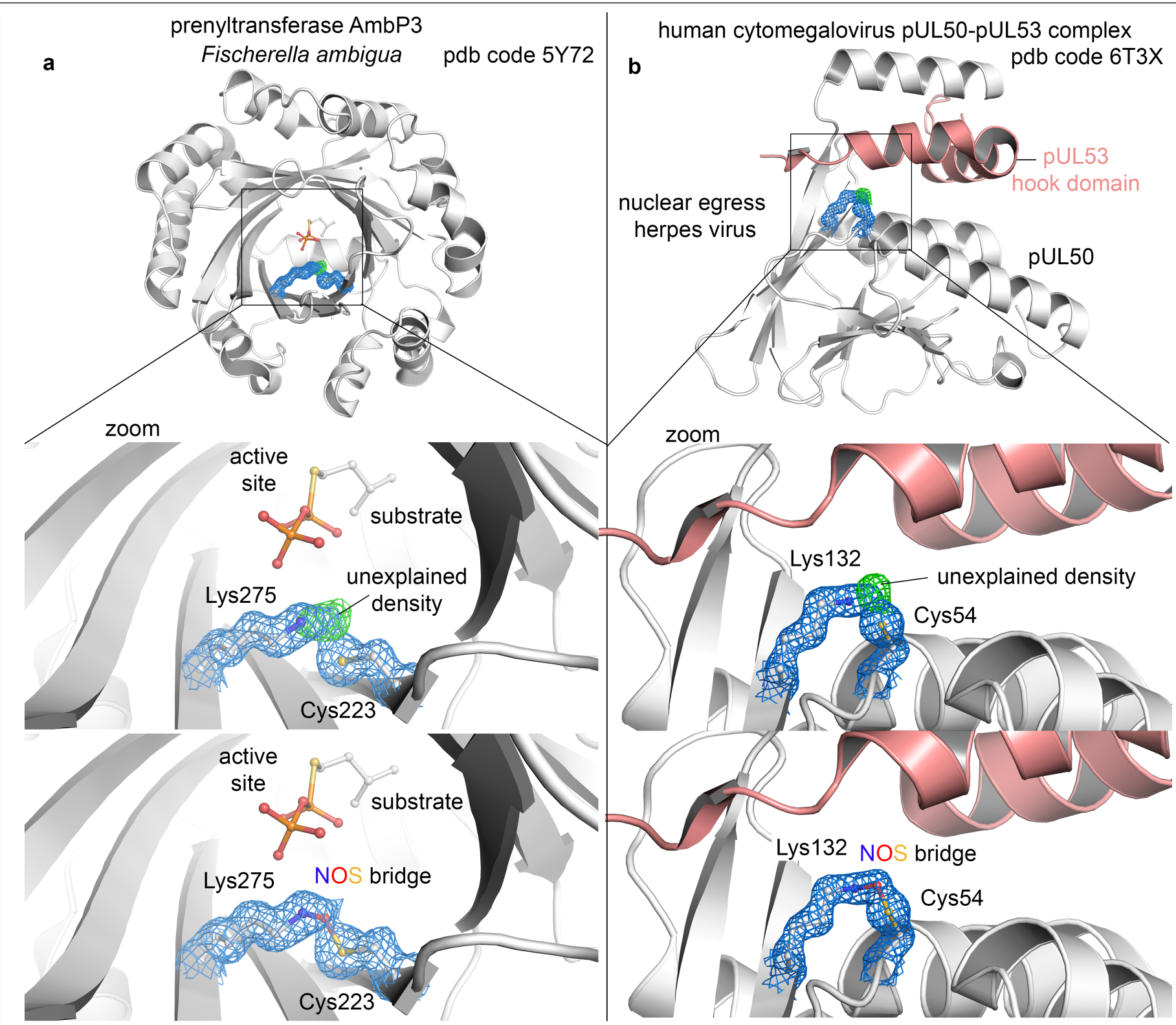

Extended Data Fig. 7 | Representative examples of protein structures deposited in the PDB that probably contain an NOS bridge akin to that of NgTAL.a, Prenyltransferase AmbP3. b, Human cytomegalovirus pUL50-pUL53 complex. For both examples, the overall structure is shown in cartoon representation in the top panel, highlighting the Lys-Cys linkage and providing the PDB code $\mathrm{e}^{56,57}$. In the corresponding middle panels, the structure of the lysine-cysteine pair as deposited in the PDB is shown enlarged, including the calculated $2 m F_{\mathrm{o}}-D F_{\mathrm{c}}$ (in blue, contour level $1 \sigma$ ) and $\mathrm{m} F_{\mathrm{o}}-D F_{\mathrm{c}}$ difference (in green, contour level $3 \sigma$ ) electron density maps. There is a pronounced positive difference peak in the electron density maps in between the lysine nitrogen atoms and the cysteine sulfur atoms, indicating the presence of a covalent bridge. In the bottom panels, the refined structural models that include the covalent lysine-cysteine NOS bridges are shown with the corresponding $2 m F-D F_{\mathrm{c}}$ electron density maps. The $m F_{\mathrm{o}}-D F_{\mathrm{c}}$ difference electron density maps are shown in green and are contoured at $3 \sigma$. The calculated occupancies of the NOS bridges amount to $62 \%$ (a) and $76 \%$ (b). The NOS bridge is prominently located at either the substrate binding site or the protein-protein binding interface. 
a

Lys 138

(catalytic lysine)

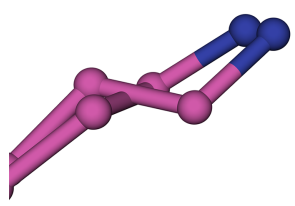

Asp17

citrate

(partially disordered)

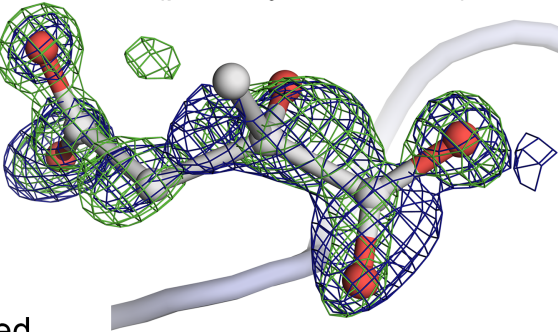

oxidized
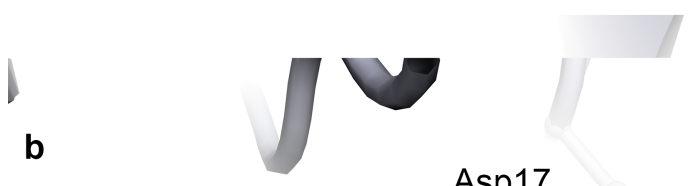

b

\section{Asp17}

Lys 138

(catalytic lysine)

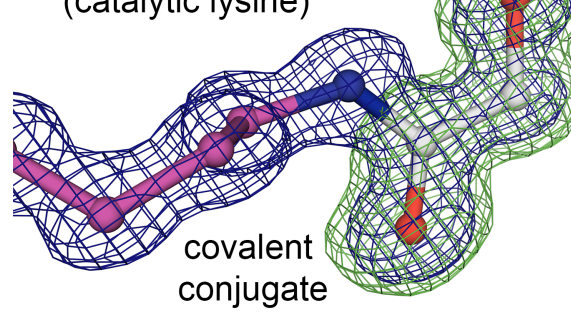

reduced
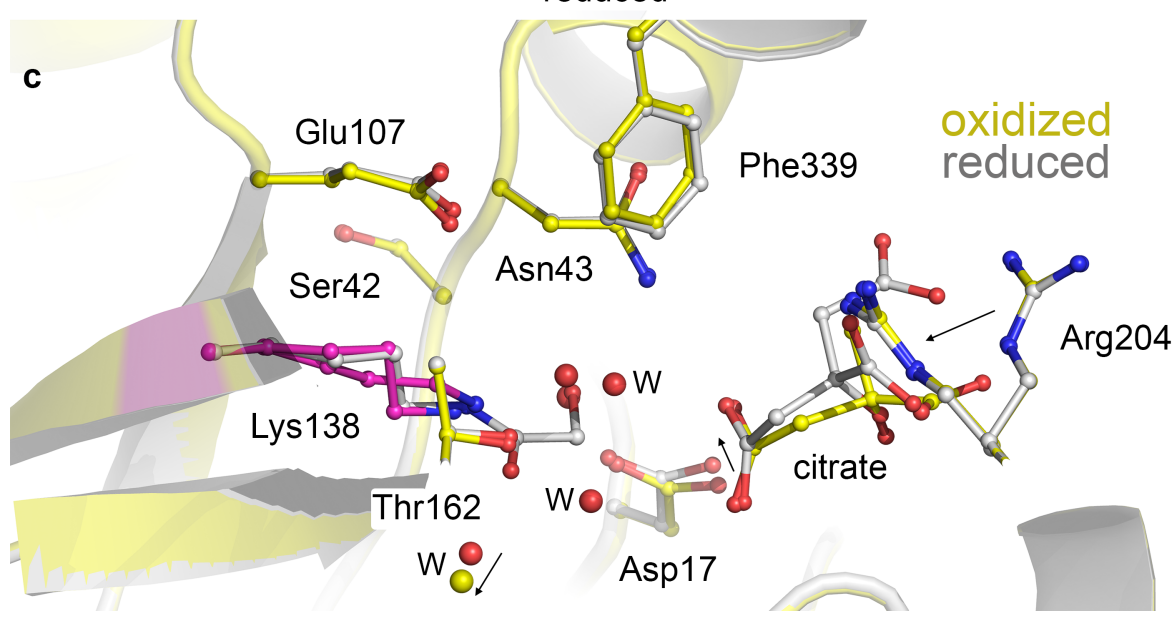

Extended Data Fig. 8 |Superposition of $\mathrm{NgTAL}$ in the oxidized and reduced state. Both of these structures are from this Article. a, Structure of the active site of $\mathrm{NgTAL}$ in the oxidized state, showing catalytic residues Lys138, Asp17 and the ligand citrate. Citrate is partially disordered. The corresponding $2 m F_{\mathrm{o}}-D F_{\mathrm{c}}$ (blue) and $m F_{\mathrm{o}}-D F_{\mathrm{c}}$ omit (green) electron density maps are shown at contour levels of $1 \sigma$ and $5 \sigma$, respectively. $\mathbf{b}$, Structure of the active site of $\mathrm{NgTAL}$ in the reduced state, showing catalytic residues Lys138, Asp17 and the ligand citrate. Citrate is structurally well-defined in this state. A covalent conjugate between Lys138 and a two-carbon fragment formed during crystallization. The corresponding $2 m F_{\mathrm{o}}-D F_{\mathrm{c}}$ (blue) and $m F_{\mathrm{o}}-D F_{\mathrm{c}}$ omit (green) electron density maps are shown at contour levels of $1 \sigma$ and $5 \sigma$, respectively. c, Superposition of the oxidized (yellow) and reduced (grey) $N g$ TAL, showing selected active site residues and the ligand citrate. The structural change of ligand citrate is accompanied by a redistribution of the two conformers of Arg204 (oxidized 70:30\% occupancy, reduced 30:70\% occupancy). There is also a subtle repositioning of the active-site residues (for example, Asp17 and water molecules). 
Extended Data Table 1 | Steady-state kinetic analysis of wild-type NgTAL and its variants, as well as of NmTAL wild type, under oxidizing and reducing conditions

\begin{tabular}{ccccc}
\hline Transaldolase & $\begin{array}{c}k_{\text {cat }} \\
{\left[\mathrm{s}^{-1}\right]}\end{array}$ & $\begin{array}{c}K_{\mathrm{M}} \\
{[\mathrm{mM}]}\end{array}$ & $\begin{array}{c}k_{\text {cat }} / K_{\mathrm{M}} \\
{\left[\mathrm{s}^{-1} \mathrm{mM}^{-1}\right]}\end{array}$ & DTT \\
\hline
\end{tabular}

\section{N. gonorrhoeae}

$\begin{array}{ccccc}\text { wt ox } & \text { steady-state } & 0.63 \pm 0.02 & 9.98 \pm 1.28 & 0.06 \\ & \text { basal } & (0.29 \pm 0.01)^{a} & (9.36 \pm 1.33)^{a} & (0.03) \\ \text { wt red } & \text { steady-state } & 17.63 \pm 0.33 & 5.62 \pm 0.41 & 3.14 \\ & & (30 \text {-fold } \pi) & (2 \text {-fold } ע) & (60 \text {-fold } \pi)\end{array}$

\begin{tabular}{|cccccc} 
Cys38Ser ox & $\begin{array}{c}\text { steady-state } \\
\text { (no activation) }\end{array}$ & $28.58 \pm 0.80$ & $3.45 \pm 0.47$ & 8.28 & - \\
\hline Cys38Ser red & steady-state & $\begin{array}{r}27.26 \pm 0.80 \\
\text { (no change) }\end{array}$ & $\begin{array}{l}3.46 \pm 0.49 \\
\text { (no change) }\end{array}$ & $\begin{array}{c}7.88 \\
\text { (no change) }\end{array}$
\end{tabular}

\begin{tabular}{|c|c|c|c|c|c|}
\hline Cys87Ala ox & $\begin{array}{c}\text { steady-state } \\
\text { basal }\end{array}$ & $\begin{array}{c}1.04 \pm 0.04 \\
(0.064 \pm 0.004)\end{array}$ & $\begin{array}{c}31.76 \pm 2.79 \\
(11.77 \pm 2.80)\end{array}$ & $\begin{array}{c}0.033 \\
(0.005)\end{array}$ & - \\
\hline Cys87Ala red & steady-state & $\begin{array}{c}13.73 \pm 0.29 \\
(15 \text {-fold } \pi)\end{array}$ & $\begin{array}{c}11.56 \pm 0.83 \\
(3-f o l d y)\end{array}$ & $\begin{array}{c}1.19 \\
(40 \text {-fold } \text { त) }\end{array}$ & + \\
\hline
\end{tabular}

$\begin{array}{cccccc}\text { Cys90Ala ox } & \text { steady-state } & 0.026 \pm 0.001 & 9.16 \pm 0.89 & 0.0028 & - \\ & \text { basal } & (0.012 \pm 0.001) & (8.26 \pm 0.89) & (0.0014) \\ \text { Cys90Ala red } & \text { steady-state } & 0.56 \pm 0.01 & 7.87 \pm 0.70 & 0.071 \\ & & (20 \text {-fold } \text { T) } & \text { (no change) } & (25 \text {-fold } \text { T) }\end{array}$

\begin{tabular}{|c|c|c|c|c|c|}
\hline Lys8Ala ox & $\begin{array}{c}\text { steady-state } \\
\text { basal }\end{array}$ & $\begin{array}{c}0.34 \pm 0.01 \\
(0.018 \pm 0.002)^{a}\end{array}$ & $\begin{array}{c}36.26 \pm 4.11 \\
(10.83 \pm 4.16)^{a}\end{array}$ & $\begin{array}{c}0.009 \\
(0.016)^{a}\end{array}$ & - \\
\hline Lys8Ala red & steady-state & $\begin{array}{c}10.20 \pm 0.17 \\
(30 \text {-fold } \pi)\end{array}$ & $\begin{array}{l}2.60 \pm 0.22 \\
(14-f o l d y)\end{array}$ & $\begin{array}{c}3.93 \\
(400-\text { fold } 7)\end{array}$ & + \\
\hline
\end{tabular}

\section{N. meningitidis}

\begin{tabular}{|c|c|c|c|c|c|}
\hline wt ox & steady-state & $21.4 \pm 0.4$ & $5.2 \pm 0.4$ & 4.11 & - \\
\hline \multirow[t]{2}{*}{ wt red } & steady-state & $48.4 \pm 0.4$ & $3.4 \pm 0.1$ & 14.24 & + \\
\hline & & (2.5-fold $\pi)$ & (2-fold ע) & (4-fold $\pi)$ & \\
\hline
\end{tabular}

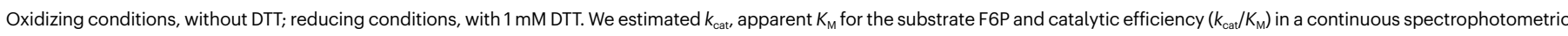

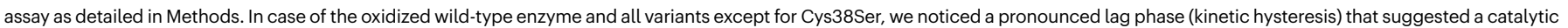

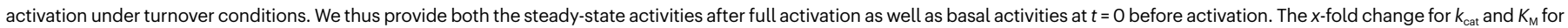

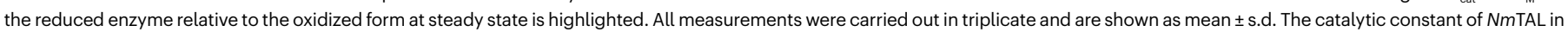
the oxidized form represents an upper limit, as oxidized and reduced species cannot be quantitatively separated by chromatographic methods as in the case of $\mathrm{NgTAL}$.

${ }^{a}$ Forcatalytic activation phases, data were analysed as described in 'Steady-state kinetic analysis' in Methods. 


\section{Article}

Extended Data Table 2 | X-ray crystallographic data collection and refinement statistics

\begin{tabular}{|c|c|c|c|c|}
\hline & $\begin{array}{c}\mathrm{NgTal} \\
\text { oxidized } \\
\text { citrate 1 }\end{array}$ & $\begin{array}{l}\mathrm{NgTAL} \\
\text { oxidized } \\
\text { citrate 2 }\end{array}$ & $\begin{array}{l}\mathrm{NgTAL} \\
\text { oxidized } \\
\text { acetate }\end{array}$ & $\begin{array}{l}\mathrm{NgTAL} \\
\text { reduced } \\
\text { citrate }\end{array}$ \\
\hline pdb code & $6 Z X 4$ & $6 Z \mathrm{WJ}$ & $6 Z \mathrm{WH}$ & $6 Z W F$ \\
\hline \multicolumn{5}{|l|}{ Data collection } \\
\hline \multicolumn{5}{|l|}{ Cell dimensions } \\
\hline $\begin{array}{l}a, b, c(\AA) \\
\alpha, \beta, Y\left({ }^{\circ}\right)\end{array}$ & $\begin{array}{l}42.2682 .7990 .40 \\
909090\end{array}$ & $\begin{array}{c}42.2082 .9590 .50 \\
909090\end{array}$ & $\begin{array}{l}42.2582 .9890 .34 \\
\quad 909090\end{array}$ & $\begin{array}{c}42.0982 .9189 .46 \\
\quad 909090\end{array}$ \\
\hline Resolution ( $(\AA)$ & $\begin{array}{c}30.53-0.96 \\
(0.99-0.96)^{\star}\end{array}$ & $\begin{array}{l}37.62-1.35 \\
(1.39-1.35)\end{array}$ & $\begin{array}{l}39.67-1.50 \\
(1.55-1.50)\end{array}$ & $\begin{array}{l}38.08-1.05 \\
(1.09-1.05)\end{array}$ \\
\hline$R_{\text {meas }}$ & $0.043(1.287)$ & $0.083(1.213)$ & $0.146(1.671)$ & $0.078(2.122)$ \\
\hline$C C_{1 / 2}$ & $0.999(0.484)$ & $0.998(0.494)$ & $0.998(0.754)$ & $0.999(0.468)$ \\
\hline$|/ \sigma|$ & $14.53(0.97)$ & $9.76(1.20)$ & $11.77(1.93)$ & $10.87(0.94)$ \\
\hline Completeness (\%) & $98.43(97.75)$ & $94.97(97.26)$ & $99.87(99.82)$ & 99.53 (99.68) \\
\hline Redundancy & $3.4(3.3)$ & $3.5(3.4)$ & $13.0(13.1)$ & $4.5(4.5)$ \\
\hline \multicolumn{5}{|l|}{ Refinement } \\
\hline Reflections used in refinement & 190383 & 67028 & 51606 & 145659 \\
\hline$R_{\text {work }} / R_{\text {free }}$ & $11.7,13.6$ & $14.6,18.2$ & $15.1,18.2$ & $13.3,15.4$ \\
\hline \multicolumn{5}{|l|}{ Number of non-hydrogen atoms } \\
\hline macromolecules & 3241 & 3110 & 3085 & 3233 \\
\hline ligands & 22 & 16 & 14 & 43 \\
\hline solvent & 535 & 459 & 397 & 496 \\
\hline \multicolumn{5}{|l|}{ Average B-factor $\left(\AA^{2}\right)$} \\
\hline macromolecules & 12.48 & 16.62 & 18.86 & 13.83 \\
\hline ligands & 19.49 & 22.12 & 29.04 & 22.07 \\
\hline solvent & 27.72 & 30.58 & 30.47 & 29.24 \\
\hline \multicolumn{5}{|l|}{ R.m.s. deviations } \\
\hline RMS (bonds) $(\AA)$ & 0.009 & 0.006 & 0.008 & 0.007 \\
\hline \multirow[t]{2}{*}{ RMS (angles) $\left({ }^{\circ}\right)$} & 1.47 & 1.2 & 1.29 & 1.27 \\
\hline & $\begin{array}{c}\mathrm{NgTal} \\
\text { oxidized } \\
\text { low-dose in house }\end{array}$ & $\begin{array}{l}\text { NgTAL } \\
\text { Lys8Ala }\end{array}$ & $\begin{array}{l}\text { NgTAL } \\
\text { Cys38Ser }\end{array}$ & \\
\hline pdb code & 7BOL & 7BBX & 7BBW & \\
\hline \multicolumn{5}{|l|}{ Data collection } \\
\hline \multicolumn{5}{|l|}{ Cell dimensions } \\
\hline $\begin{array}{l}a, b, c(\AA) \\
\alpha, \beta, y\left({ }^{\circ}\right)\end{array}$ & $\begin{array}{l}42.1582 .9189 .53 \\
\quad 909090\end{array}$ & $\begin{array}{c}42.4282 .8990 .25 \\
909090\end{array}$ & $\begin{array}{c}44.9753 .99153 .86 \\
9094.04990\end{array}$ & \\
\hline Resolution ( $\AA$ ) & $\begin{array}{c}38.14-1.65 \\
(1.71-1.65)^{*}\end{array}$ & $\begin{array}{l}37.76-0.85 \\
(0.88-0.85)\end{array}$ & $\begin{array}{l}32.61-1.25 \\
(1.29-1.25)\end{array}$ & \\
\hline$R_{\text {meas }}$ & $0.092(0.816)$ & $0.051(2.198)$ & $0.037(0.493)$ & \\
\hline$C C_{1 / 2}$ & $0.998(0.598)$ & $0.998(0.494)$ & $0.999(0.821)$ & \\
\hline$\| \sigma /$ & $15.33(1.44)$ & $23.84(1.12)$ & $20.86(3.28)$ & \\
\hline Completeness (\%) & $96.22(80.45)$ & $99.18(95.30)$ & $98.13(98.55)$ & \\
\hline Redundancy & $7.3(2.1)$ & $12.8(10.1)$ & $3.5(3.4)$ & \\
\hline \multicolumn{5}{|l|}{ Refinement } \\
\hline Reflections used in refinement & 37095 & 276267 & 199408 & \\
\hline$R_{\text {work }} / R_{\text {free }}$ & $15.1,19.2$ & $10.8,11.9$ & $13.0,15.2$ & \\
\hline \multicolumn{5}{|l|}{ Number of non-hydrogen atoms } \\
\hline macromolecules & 2693 & 3173 & 5766 & \\
\hline ligands & 13 & 17 & 6 & \\
\hline solvent & 461 & 537 & 1226 & \\
\hline Average B-factor $\left(\AA^{2}\right)$ & 15.46 & 13.02 & 18.35 & \\
\hline macromolecules & 13.36 & 10.88 & 16.1 & \\
\hline ligands & 16.11 & 11.42 & 33.23 & \\
\hline solvent & 27.71 & 25.72 & 28.88 & \\
\hline \multicolumn{5}{|l|}{ R.m.s. deviations } \\
\hline RMS (bonds) (Å) & 0.008 & 0.008 & 0.007 & \\
\hline RMS (angles) $\left({ }^{\circ}\right)$ & 1.02 & 1.14 & 0.99 & \\
\hline
\end{tabular}

*Values in parentheses are for the highest-resolution shell. 


\section{Reporting Summary}

Nature Research wishes to improve the reproducibility of the work that we publish. This form provides structure for consistency and transparency in reporting. For further information on Nature Research policies, see our Editorial Policies and the Editorial Policy Checklist.

\section{Statistics}

For all statistical analyses, confirm that the following items are present in the figure legend, table legend, main text, or Methods section.

n/a Confirmed

$\square$ The exact sample size $(n)$ for each experimental group/condition, given as a discrete number and unit of measurement

$\square$ \A statement on whether measurements were taken from distinct samples or whether the same sample was measured repeatedly

$\triangle$ The statistical test(s) used AND whether they are one- or two-sided

$\triangle$ Only common tests should be described solely by name; describe more complex techniques in the Methods section.

Х $\square$ A description of all covariates tested

Х $\square$ A description of any assumptions or corrections, such as tests of normality and adjustment for multiple comparisons

$\triangle$ A full description of the statistical parameters including central tendency (e.g. means) or other basic estimates (e.g. regression coefficient)

$\bigotimes$ AND variation (e.g. standard deviation) or associated estimates of uncertainty (e.g. confidence intervals)

X For null hypothesis testing, the test statistic (e.g. $F, t, r$ ) with confidence intervals, effect sizes, degrees of freedom and $P$ value noted

Qive $P$ values as exact values whenever suitable.

Х $\square$ For Bayesian analysis, information on the choice of priors and Markov chain Monte Carlo settings

Х $\square$ For hierarchical and complex designs, identification of the appropriate level for tests and full reporting of outcomes

Х $\square$ Estimates of effect sizes (e.g. Cohen's $d$, Pearson's $r$ ), indicating how they were calculated

Our web collection on statistics for biologists contains articles on many of the points above.

\section{Software and code}

Policy information about availability of computer code

Data collection Kinetics and spectroscopic analysis

Chirascan Spectrometer Control Panel Application version 4.2.27 (Circular Dichroism)

Spectra Manager version 2.07.02 (Build 4) (UV-Vis-based Kinetics)

X-ray crystallography

mxCUBE 2 (X-ray data collection at P14 DESY/EMBL, Hamburg, Germany)

Mass spectrometry

Orbitrap Exploris 480 or Q Exactive (Thermo Scientific), both coupled to a Dionex UltiMate 3000 UHPLC system (Thermo Scientific).

Analytical ultracentrifugation

ProteomeLab XL-I, GUI 6.0 (Firmware 5.7)

Electronic structure calculations

Gaussian16

Data analysis Kinetics and spectroscopic analysis

Pro-Data Viewer version 4.2.27 (Circular Dichroism)

Spectra Manager version 2.07.02 (Build 4) (UV-Vis-based Kinetics)

SigmaPlot version 11.0 (Circular Dichroism, UV-Vis-based Kinetics)

Crystallography

XDS VERSION Mar 15, 2019 BUILT=20190315 (X-ray, data processing)

XSCALE VERSION Jan 31, 2020 BUILT=20200417 (X-ray, data scaling) 
CCP4 version 7.0.78 (X-ray, processing and refinement)

phenix.refine version 1.13_2998 (structure, refinement)

COOT version 0.8.9.2 (structure, model building)

MolProbity-Server Version 4 (structure validation)

The PyMOL Molecular Graphics System version 1.3 (structure representation)

Electronic structure calculations

Gaussian16

Python3.1 (libraries: numpy)

Mass spectrometry data analysis

Freestyle 1.3

MaxQuant 1.6.5.0

Analytical ultracentrifugation

SEDFIT version $16.2 \mathrm{~b}$ (fitting of the raw data with the Continuous $\mathrm{c}(\mathrm{s})$ Distribution model)

SEDNTERP version 1.09 (calculation of extinction coefficients, partial specific volumes, density, viscosity, s 20, w correction)

GUSSI version 1.4 .6 (preparation of figures)

Phylognetic analysis

MAFFT 7

iTOL 5

Jalview 2.11

For manuscripts utilizing custom algorithms or software that are central to the research but not yet described in published literature, software must be made available to editors and reviewers. We strongly encourage code deposition in a community repository (e.g. GitHub). See the Nature Research guidelines for submitting code \& software for further information.

\section{Data}

Policy information about availability of data

All manuscripts must include a data availability statement. This statement should provide the following information, where applicable:

- Accession codes, unique identifiers, or web links for publicly available datasets

- A list of figures that have associated raw data

- A description of any restrictions on data availability

The refined structural protein models and corresponding structure-factor amplitudes are deposited under PDB accession codes 6XZ4 (NgTAL oxidized citrate 1), 6ZWJ (NgTAL oxidized citrate 2), 6ZWH (NgTAL oxidized acetate), 6ZWF (NgTAL reduced citrate), 7BOL (NgTAL oxidized low-dose), 7BBX (NgAL variant Lys8Ala) and 7BBW (NgTAL variant Cys38Ser). The structures cited in this publication (1M3Q, 3CLM, 6T3X, 5Y72) are available under their respective PDB accession codes. All other data are available on request.

\section{Field-specific reporting}

Please select the one below that is the best fit for your research. If you are not sure, read the appropriate sections before making your selection. \ Life sciences $\quad \square$ Behavioural \& social sciences $\quad \square$ Ecological, evolutionary \& environmental sciences For a reference copy of the document with all sections, see nature.com/documents/nr-reporting-summary-flat.pdf

\section{Life sciences study design}

All studies must disclose on these points even when the disclosure is negative.

Sample size n.a.

Data exclusions n.a.

Replication Measurements were repeated at least two times.

Randomization n.a.

Blinding n.a.

\section{Reporting for specific materials, systems and methods}

We require information from authors about some types of materials, experimental systems and methods used in many studies. Here, indicate whether each material, system or method listed is relevant to your study. If you are not sure if a list item applies to your research, read the appropriate section before selecting a response. 
Materials \& experimental systems Methods

\begin{tabular}{l|l}
\hline$n / a$ & Involved in the study \\
$\square$ & Antibodies \\
$\square$ Eukaryotic cell lines \\
$\square$ Palaeontology and archaeology \\
$\square$ & $\square$ Animals and other organisms \\
$\square$ & $\square$ Clinical data
\end{tabular}

$\mathrm{n} / \mathrm{a}$ Involved in the study

\ $\square$ ChIP-seq

$\bigotimes \square$ Flow cytometry

$\bigotimes \mid \square$ MRI-based neuroimaging

X $\square$ Dual use research of concern 